CUBO A Mathematical Journal

Vol.14, No 02, (91-109). June 2012

\title{
Higher order terms for the quantum evolution of a Wick observable within the Hepp method
}

\author{
SÉBASTIEN BRETEAuX \\ IRMAR, UMR-CNRS 6625, Université de Rennes 1, campus de Beaulieu, \\ 35042 Rennes Cedex, France. \\ ENS de Cachan, Antenne de Bretagne, Campus de Ker Lann, Av. R. Schuman, \\ 35170 Bruz, France. \\ email: sebastien.breteaux@ens-cachan.org
}

\begin{abstract}
The Hepp method is the coherent state approach to the mean field dynamics for bosons or to the semiclassical propagation. A key point is the asymptotic evolution of Wick observables under the evolution given by a time-dependent quadratic Hamiltonian. This article provides a complete expansion with respect to the small parameter $\varepsilon>0$ which makes sense within the infinite-dimensional setting and fits with finite-dimensional formulae.
\end{abstract}

\section{RESUMEN}

El método de Hepp describe en términos de estados coherentes la dinámica en campo medio de bosones o la propagación semiclásica. Un punto clave es la evolución asintótica de observables de Wick bajo la evolución dada por un Hamiltoniano cuadrático dependiente del tiempo. Este artículo proporciona una expansión completa con respecto al parámetro pequeño $\varepsilon>0$ válido en dimensión infinita y que corresponde a fórmulas en dimensión finita conocidas.

Keywords and Phrases: Mean field limit, semiclassical limit, coherent states, squeezed states.

2000 AMS Mathematics Subject Classification: 81R30, 35Q40, 81S10, 81S30. 


\section{Introduction}

In this article we derive two expansions with respect to a small parameter $\varepsilon$ of quantum evolved Wick observables under a time-dependent quadratic Hamiltonian.

The Hepp method was introduced in [20] and then extended in [12, 13] in order to study the mean field dynamics of many bosons systems via a (squeezed) coherent states approach. The asymptotic analysis in the mean field limit is done with respect to a small parameter $\varepsilon$, where the number of particles is of order $\frac{1}{\varepsilon}$.

Remember that the mean field dynamics is obtained as a classical Hamiltonian dynamics which governs the evolution of the center $z(t)$ of the Gaussian state (squeezed coherent state). Meanwhile the covariance of this Gaussian as well as the control of the remainder term is determined by the evolution of a quadratic approximate Hamiltonian around $z(t)$.

A key point in this method is the asymptotic analysis of the evolution of a Wick quantized observable according to this quantum time-dependent quadratic Hamiltonian.

Only a few results are clearly written about the remainder terms and some possible expansions in powers of $\varepsilon$, see the works of Ginibre and Velo [14, 15]. In the finite-dimensional case, entering into the semiclassical theory, accurate results have been given by Combescure, Ralston and Robert in [6, 7, 8, Hagedorn and Joye in [17, 18, 19. Another viewpoint is used (in finite dimension) by Paul and Uribe in 25] to get approximate eigenvectors of semiclassical operators in terms of linear superpositions of coherent states. For the mean field infinite-dimensional setting some results have been proved in [16, 11, 28] with a different approach.

We stick here with the Hepp method with the presentation of [1] which puts the stress on the similarities and differences between the infinite-dimensional bosonic mean field problem and the finite-dimensional semiclassical analysis. Nevertheless, in [1] the authors only considered the main order term although some of their formulae make possible complete expansions. In this article we derive two expansions of the quantum evolved Wick observables which are equal term by term.

Two difficulties have to be solved :

(1) Unlike the time-independent finite-dimensional case, no Mehler type explicit formula (see for example [22] or 9]) is available. A general time-dependent Hamiltonian has no explicit dynamics.

(2) In the infinite-dimensional framework the quantization of a linear symplectic transformation (a Bogoliubov transformation) requires some care. Useful references on this subject are [3] and [2]. Its realization in the Fock space relies on a Hilbert-Schmidt condition on the antilinear part connected with the Shale theorem (see [29] and [26, 8, 5]).

These things are well known but have to be considered accurately while writing complete expansions. 
Two different methods, with apparently two different final formulae, will be used. A first one relies on a Dyson expansion approach and provides the successive terms as time-dependent integrals. The second one uses the exact formulae for the finite-dimensional Weyl quantization and after having made explicit the relationship between Wick and Weyl quantizations like in [4] or [1, the proper limit process with respect to the dimension is carried out.

The outline of this article is the following. In Section 2 we recall some facts and definitions about the Fock space and Wick quantization. We then present our main results in Section 3 in Theorems 3.1 and 3.2 and illustrate them by a simple example. Section 4 and Section 5 are devoted to the construction and properties of the classical and quantum evolution associated with a symmetric quadratic Hamiltonian. Section 7 and Section 8 contain the proofs of our two expansion formulae. For the convenience of the reader we recall some facts about real-linear symplectomorphisms and symplectic Fourier transform in the appendices.

\section{Wick calculus with polynomial observables}

\subsection{Definitions}

We recall some definitions and results about Wick quantization. More details can be found in [1]

In this paper $(\mathcal{Z},\langle\cdot, \cdot\rangle)$ denotes a separable Hilbert space over $\mathbb{C}$, the field of complex numbers. It is also a symplectic space with respect to the symplectic form $\sigma\left(z_{1}, z_{2}\right)=\mathfrak{I}\left\langle z_{1}, z_{2}\right\rangle$. We use the physicists convention that all the scalar products over Hilbert spaces are linear with respect to the right variable and antilinear with respect to the left variable. We denote by $\mathcal{S}_{\mathrm{m}}$ the symmetrization operator on $\otimes^{m} \mathcal{Z}$ (the completion for the natural Hilbert scalar product of the algebraic tensor

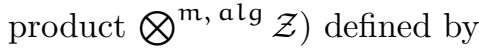

$$
\mathcal{S}_{\mathrm{m}}\left(z_{1} \otimes \cdots \otimes z_{\mathrm{m}}\right)=\frac{1}{\mathrm{~m} !} \sum_{\sigma \in \mathfrak{S}_{\mathrm{m}}} z_{\sigma_{1}} \otimes \cdots \otimes z_{\sigma_{\mathrm{m}}}
$$

where the $z_{j}$ are vectors in $\mathcal{Z}$ and $\mathfrak{S}_{\mathfrak{m}}$ denotes the set of the permutations of $\{1, \ldots, \mathfrak{m}\}$. We will use the notation $z_{1} \vee \cdots \vee z_{\mathrm{m}}$ for $\mathcal{S}_{\mathrm{m}}\left(z_{1} \otimes \cdots \otimes z_{\mathrm{m}}\right)$, and $z^{\vee \mathrm{m}}$ for $z \vee \cdots \vee z$ when the $\mathrm{m}$ terms of this product are equal to $z$. We call monomial of order $(p, q) \in \mathbb{N}^{2}$ a complex-valued application defined on $\mathcal{Z}$ of the form

$$
\mathrm{b}(z)=\left\langle z^{\vee \mathrm{q}}, \tilde{\mathrm{b}} z^{\vee p}\right\rangle
$$

with $\widetilde{\mathrm{b}} \in \mathcal{L}\left(\bigvee^{\mathfrak{p}} \mathcal{Z}, \bigvee^{\mathrm{q}} \mathcal{Z}\right)$ where $\bigvee^{\mathrm{n}} \mathcal{Z}$ (or $\mathcal{Z}^{\vee n}$ ) denotes the Hilbert completion of the $\mathrm{n}$-fold symmetric tensor product, and for two Banach spaces $E$ and $F$, the space of continuous linear applications from $E$ to $F$ is denoted by $\mathcal{L}(E, F)$. We then write $b \in \mathcal{P}_{p, q}(\mathcal{Z})$. The total order of $b$ is the integer $m=p+q$. The finite linear combinations of monomials are called polynomials. The set of all polynomials of this type is denoted by $\mathcal{P}(\mathcal{Z})$. Subsets of particular interest of $\mathcal{P}(\mathcal{Z})$ are $\mathcal{P}_{\mathrm{m}}(\mathcal{Z})$ and $\mathcal{P}_{\leq \mathrm{m}}(\mathcal{Z})$, the finite linear combinations of monomials of total order equal to $\mathrm{m}$ and not greater than $\mathrm{m}$. 
The Hilbert space

$$
\mathcal{H}:=\bigoplus_{n \in \mathbb{N}} \bigvee^{n} \mathcal{Z}
$$

is called the symmetric Fock space associated with $\mathcal{Z}$, where tensor products and sum completions are made with respect to the natural Hilbert scalar products inherited from $\mathcal{Z}$. We also consider the dense subspace $\mathcal{H}_{\text {fin }}$ of $\mathcal{H}$ of states with a finite number of particles

$$
\mathcal{H}_{\text {fin }}:=\bigoplus_{n \in \mathbb{N}}^{\operatorname{alg}} \bigvee^{n} \mathcal{Z}
$$

where the tensor products are completed but the sum is algebraic.

The Wick quantization of a monomial $\mathrm{b} \in \mathcal{P}_{\mathrm{p}, \mathrm{q}}(\mathcal{Z})$ is the operator defined on $\mathcal{H}_{\text {fin }}$ by its action on $\bigvee^{\mathrm{n}} \mathcal{Z}$ as an element of $\mathcal{L}\left(\bigvee^{\mathrm{n}} \mathcal{Z}, \bigvee^{\mathrm{n}+\mathrm{q}-\mathrm{p}} \mathcal{Z}\right)$

$$
\left.\mathrm{b}^{\text {Wick }}\right|_{\vee^{n} \mathcal{Z}}=1_{[p,+\infty)}(n) \frac{\sqrt{n !(n+q-p) !}}{(n-p) !} \varepsilon^{\frac{p+q}{2}}\left(\tilde{b} \vee I_{\vee^{n-p} \mathcal{Z}}\right)
$$

where $I_{X}$ denotes the identity map on the space $X$ and for $A_{j} \in \mathcal{L}\left(\mathcal{Z}^{\vee p_{j}}, \mathcal{Z}^{\vee q_{j}}\right), A_{1} \vee A_{2}=$ $\mathcal{S}_{\mathrm{q}_{1}+\mathrm{q}_{2}} A_{1} \otimes A_{2} \mathcal{S}_{\mathrm{p}_{1}+\mathrm{p}_{2}}$. The Wick quantization is extended by linearity to polynomials.

We have a notion of derivative of a polynomial, first defined on the monomials and then extended by linearity. For $\mathrm{b} \in \mathcal{P}_{\mathrm{p}, \mathrm{q}}(\mathcal{Z})$ and for any given $z \in \mathcal{Z}$, the operator

$$
\partial_{\bar{z}}^{j} \partial_{z}^{k} b(z):=\frac{p !}{(p-k) !} \frac{q !}{(q-j) !}\left(\left\langle z^{\vee(q-j)}\right| \vee I_{\bigvee^{j} \mathcal{Z}}\right) \tilde{b}\left(z^{\vee(p-k)} \vee I_{\bigvee^{k} \mathcal{Z}}\right)
$$

is an element of $\mathcal{L}\left(\bigvee^{k} \mathcal{Z}, \bigvee^{j} \mathcal{Z}\right)$. We use the "bra" and "ket" notations of the physicists for vectors and forms in Hilbert spaces. Then we can define the Poisson bracket of order $k$ of two polynomials $b_{1}, b_{2}$, by

$$
\left\{b_{1}, b_{2}\right\}^{(k)}=\partial_{z}^{k} b_{1} \cdot \partial_{\bar{z}}^{k} b_{2}-\partial_{z}^{k} b_{2} \cdot \partial_{\bar{z}}^{k} b_{1}
$$

since, for any polynomial $b, \partial_{z}^{k} b(z)$ is a $k$-form (on $\mathcal{Z}$ ) and $\partial_{\bar{z}}^{k} b(z)$ is a k-vector.

Remark 1. The product denoted by a dot in the definition of the Poisson bracket is a $\mathbb{C}$-bilinear duality-product between k-forms and k-vectors. As an example consider the polynomials

$$
\mathrm{b}_{1}(z)=\left\langle z^{\vee 3}, \xi_{1}^{\vee 3}\right\rangle\left\langle\eta_{1}^{\vee 2}, z^{\vee 2}\right\rangle \quad \text { and } \quad b_{2}(z)=\left\langle z^{\vee 3}, \xi_{2}^{\vee 3}\right\rangle\left\langle\eta_{2}, z\right\rangle
$$

The Poisson bracket of order 2 of $b_{1}$ and $b_{2}$ is

$$
\left\{\mathrm{b}_{1}, \mathrm{~b}_{2}\right\}^{(2)}(z)=2 \times 6 \times\left\langle z^{\vee 3}, \xi_{1}^{\vee 3}\right\rangle\left\langle\eta_{1}^{\vee 2} \vee z, \xi_{2}^{\vee 3}\right\rangle\left\langle\eta_{2}, z\right\rangle-0
$$

\subsection{Some examples of Wick quantizations}

Here is a quick review of the notations used for some useful examples of Wick quantization. A vector of $\mathcal{Z}$ is denoted by $\xi, A$ is a bounded operator and $z$ is the variable of the polynomials. In 
the next table, the first column describes the polynomial and the second the corresponding Wick quantization (as an operator on $\mathcal{H}_{\text {fin }}$ ).

$$
\begin{array}{rlc}
\langle z, A z\rangle & \leftrightarrow & \mathrm{d} \Gamma(\mathrm{A}) \\
|z|^{2} & \leftrightarrow & \mathrm{N} \\
\langle z, \xi\rangle & \leftrightarrow & \mathrm{a}^{*}(\xi) \\
\langle\xi, z\rangle & \leftrightarrow & \mathrm{a}(\xi) \\
\sqrt{2} \mathfrak{\Re}\langle z, \xi\rangle & \leftrightarrow & \Phi(\xi)
\end{array}
$$

The operator $\mathrm{d} \Gamma(\mathrm{A})$ is the usual second quantization of an operator restricted to $\mathcal{H}_{\mathrm{fin}}$ multiplied by a factor $\varepsilon$. If $A=I_{\mathcal{Z}}$ we obtain $N$ the usual number operator multiplied by a factor $\varepsilon$. The operators $a, a^{*}$ and $\Phi$ are the usual annihilation, creation and field operators of quantum field theory with an additional $\sqrt{\varepsilon}$ factor. The real and imaginary parts of a complex number $\zeta$ are denoted by $\mathfrak{R} \zeta$ and $\Im \zeta$. The field operators $\Phi(\xi)$ are essentially self-adjoint and this enables us to define the (E-dependent) Weyl operators

$$
W(\xi)=e^{i \Phi(\xi)}
$$

\subsection{Calculus}

Here are some calculation rules for Wick quantization of polynomials in $\mathcal{P}(\mathcal{Z})$. The proofs can be found in [1].

Proposition 2.1. For every polynomial $\mathrm{b} \in \mathcal{P}(\mathcal{Z})$,

- $b_{1}^{W i c k} b_{2}^{W i c k}=\left(\sum_{k=0}^{\min \left\{p_{1}, q_{2}\right\}} \frac{\varepsilon^{k}}{k !} \partial_{z}^{k} b_{1} . \partial_{\bar{z}}^{k} b_{2}\right)^{\text {Wick }}$ in $\mathcal{H}_{\text {fin }}$ for any $b_{i} \in \mathcal{P}_{p_{i}, q_{i}}(\mathcal{Z})$,

- $\mathrm{b}^{\text {Wick }}$ is closable and the domain of the closure contains

$$
\mathcal{H}_{0}=\operatorname{Vect}\left\{\mathrm{W}(z) \varphi, \varphi \in \mathcal{H}_{\text {fin }}, z \in \mathcal{Z}\right\}
$$

(we still denote by $b^{\text {Wick }}$ the closure of $b^{\text {Wick }}$ ),

- $\left(\mathrm{b}^{\text {Wick}}\right)^{*}=\overline{\mathrm{b}}^{\text {Wick }}$ on $\mathcal{H}_{\mathrm{fin}}$ (where the bar denotes the usual conjugation on complex numbers),

- for any $z_{0}$ in $\mathcal{Z}, W\left(\frac{\sqrt{2}}{i \varepsilon} z_{0}\right)^{*} b^{W i c k} W\left(\frac{\sqrt{2}}{i \varepsilon} z_{0}\right)=\left(b\left(z_{0}+z\right)\right)^{\text {Wick }}$ holds on $\mathcal{H}_{0}$ where $b\left(z_{0}+\cdot\right) \in$ $\mathcal{P}(\mathcal{Z})$.

\section{Main results and a simple example}

Our two hypotheses are: 
H1 Let $\left(\alpha_{t}\right)_{t \in \mathbb{R}}$ be a one parameter family of self-adjoint operators on $\mathcal{Z}$ defining a strongly continuous dynamical system $\mathfrak{u}_{\alpha}(t, s)$.

H1' Assume $\mathbf{H 1}$ and additionally that the dynamical system preserves a dense set D such that, for any $\psi \in \mathrm{D}, \mathfrak{u}_{\alpha}(\cdot, \cdot) \psi$ belongs to $\mathcal{C}^{1}\left(\mathbb{R}^{2}, \mathcal{Z}\right) \cap \mathcal{C}^{0}\left(\mathbb{R}^{2}, \mathrm{D}\right)$.

H2 Let $\beta$ be in $\mathcal{C}^{0}\left(\mathbb{R} ; \mathcal{Z}^{\vee 2}\right)$, ( $\beta_{\mathrm{t}}$ defines a $\mathbb{C}$-antilinear Hilbert-Schmidt operator by $\left.z \mapsto\left(\mathrm{I}_{\mathcal{Z}} \vee\langle z|\right) \beta_{\mathrm{t}}\right)$.

With $\mathbf{H 1}$ ' and $\mathbf{H 2}$, the classical flow associated with a family $\mathrm{Q}_{\mathrm{t}}(z)=\left\langle z, \alpha_{\mathrm{t}} z\right\rangle+\mathfrak{I}\left\langle\beta_{\mathrm{t}}, z^{\vee 2}\right\rangle$ of quadratic polynomials is the solution $\varphi(t, s)$ to the equation

$$
\left\{\begin{aligned}
i \partial_{t} \varphi(t, 0)[z] & =\partial_{\bar{z}} Q_{t}(\varphi(t, 0)[z]) \\
\varphi(0,0) & =I_{\mathcal{Z}}
\end{aligned}\right.
$$

where $\partial_{\bar{z}} Q_{t}(z)=\alpha z+i\left(I_{\mathcal{Z}} \vee\langle z| \beta\right)$, written in a weak sense.

Although things are better visualized by writing a differential equation, the hypotheses $\mathbf{H 1}$ and H2 suffice to define the dynamical system $\varphi(t, s)$. Details about this point are given in Section 4 Actually $\varphi(t, s)$ is a family of symplectomorphisms of $(\mathcal{Z}, \sigma)$ which are naturally decomposed into their $\mathbb{C}$-linear and $\mathbb{C}$-antilinear parts:

$$
\varphi=\mathrm{L}+\mathrm{A}, \quad \mathrm{L} \in \mathcal{L}(\mathcal{Z}), \quad \mathrm{AA}^{*} \in \mathcal{L}_{1}(\mathcal{Z})
$$

See Appendix $\mathrm{A}$ for more details about symplectomorphisms and this decomposition.

Similarly, the quantum flow associated with $\mathrm{Q}_{\mathrm{t}}$ is the solution $\mathrm{U}(\mathrm{t}, \mathrm{s})$ to

$$
\left\{\begin{array}{rl}
i \varepsilon \partial_{t} U(t, 0) & =Q_{t}^{\text {Wick }} U(t, 0) \\
U(0,0) & =I_{\mathcal{H}}
\end{array} .\right.
$$

The precise meaning of the solutions to this equation is specified in Section 5 ,

We are ready to state our two main results dealing with the evolution of a Wick observable $b^{\text {Wick }}, b \in \mathcal{P}(\mathcal{Z})$, under the quantum flow, that is to say the quantity $u(0, t) b^{\text {Wick }} u(t, 0)$. (We use the usual notation $\langle\mathrm{N}\rangle=\sqrt{\mathrm{N}^{2}+1}$.)

Theorem 3.1. Assume $\boldsymbol{H} 1$ and $\boldsymbol{H} 2$. Let $\mathrm{b} \in \mathcal{P}_{\leq m}(\mathcal{Z})$ be a polynomial. Then, for any time $\mathrm{t} \geq 0$, the formula

$$
\mathrm{U}(0, \mathrm{t}) \mathrm{b}^{\text {Wick }} \mathrm{u}(\mathrm{t}, 0)=\left(\mathrm{b}^{(0), t}\right)^{\text {Wick }}+\sum_{k=1}^{\lfloor m / 2\rfloor}\left(\frac{\varepsilon}{2}\right)^{k} \int_{\Delta_{t}^{k}}\left(b^{(k) t, \bar{s}^{k}}\right)^{W i c k} d \bar{s}^{k}
$$

holds as an equality of continuous operators from $\mathcal{D}\left(\langle\mathrm{N}\rangle^{\mathrm{m} / 2}\right)$ to $\mathcal{H}$, where

- $\bar{s}^{k}=\left(s_{1}, \ldots, s_{k}\right) \in \mathbb{R}_{+}^{k}$ and $\Delta_{t}^{k}=\left\{\bar{s}^{k} \in \mathbb{R}_{+}^{k}, \sum_{j=1}^{k} s_{j} \leq t\right\}$, 
- the polynomials $\mathrm{b}^{(\mathrm{k}) \mathrm{t}, \overline{\mathrm{s}}^{\mathrm{k}}}$ are defined recursively by

$$
\left\{\begin{aligned}
b^{(0) t}(z) & =b(\varphi(t, 0) z) \\
b^{(k+1) t, \bar{s}^{k+1}} & =\lambda^{s_{k+1}} b^{(k) t, \bar{s}^{k}},
\end{aligned}\right.
$$

with $\lambda^{s} \mathrm{c}=-\mathrm{i}\left\{\mathrm{c} \circ \varphi(0, s), \mathrm{Q}_{s}\right\}^{(2)} \circ \varphi(\mathrm{s}, 0)$ for any polynomial $c$.

Theorem 3.2. Assume $\boldsymbol{H} 1$ and $\boldsymbol{H}$ 2. Let $\mathrm{m} \geq 2$ and $\mathrm{b} \in \mathcal{P}_{\leq \mathrm{m}}(\mathcal{Z})$ a polynomial. Then introducing

- the vector $v_{\mathrm{t}} \in \bigotimes^{2} \mathcal{Z}$ such that for all $z_{1}, z_{2} \in \mathcal{Z}$,

$$
\left\langle z_{1} \otimes z_{2}, v_{\mathrm{t}}\right\rangle=\left\langle z_{1}, \mathrm{~L}^{*}(\mathrm{t}, 0) A(\mathrm{t}, 0) z_{2}\right\rangle,
$$

- the operator on $\mathcal{P}(\mathcal{Z})$

$$
\Lambda^{t} \mathrm{c}(z)=\operatorname{Tr}\left[-2 A^{*}(t, 0) A(t, 0) \partial_{\bar{z}} \partial_{z} c(z)\right]+\left\langle v_{t}\left|\cdot \partial_{\bar{z}}^{2} c(z)+\partial_{z}^{2} c(z) \cdot\right| v_{t}\right\rangle
$$

the formula

$$
u(0, t) b^{W i c k} u(t, 0)=\left(e^{\frac{\varepsilon}{2} \Lambda^{t}}(b \circ \varphi(t, 0))\right)^{\text {Wick }}
$$

holds as an equality of continuous operators from $\mathcal{D}\left(\langle\mathrm{N}\rangle^{\mathrm{m} / 2}\right)$ to $\mathcal{H}$.

Remark 2. The derivative $\partial_{\bar{z}} \partial_{z} c(z)$ is in $\mathcal{L}(\mathcal{Z})$ and $\operatorname{Tr}$ denotes the trace on the subset of trace class operators of $\mathcal{L}(\mathcal{Z})$.

Remark 3. For $\mathrm{m} \geq 2$ the operators $\lambda^{\mathrm{t}}$ and $\Lambda^{\mathrm{t}}$ send $\mathcal{P}_{\mathrm{m}}(\mathcal{Z})$ into $\mathcal{P}_{\mathrm{m}-2}(\mathcal{Z})$.

Remark 4. The exponential is intended in the sense

$$
e^{\frac{\varepsilon}{2} \Lambda^{t}} b=\sum_{k=0}^{\lfloor m / 2\rfloor} \frac{1}{k !}\left(\frac{\varepsilon}{2} \Lambda^{t}\right)^{k} b
$$

for a polynomial $b$ in $\mathcal{P}_{\leq m}(\mathcal{Z})$.

Example 1. To give an idea of the behavior of these formulae we apply them in the simplest (non trivial) possible situation, with $\mathcal{Z}=\mathbb{C}$ and $Q_{\mathrm{t}}(z)=\mathfrak{I}\left(z^{2}\right)$. As $\mathrm{Q}_{\mathrm{t}}$ is time-independent the classical evolution equation is autonomous and thus we can write $\varphi(t, s)=\varphi(t-s)$ and $i \partial_{t} \varphi(t) z=$ $\partial_{\bar{z}} \mathrm{Q}(\varphi(\mathrm{t}) z)=i \overline{\varphi(t) z}$. The solution is $\varphi(t) z=z \cosh t+\bar{z} \sinh t$. We can then compute both

$$
\int_{0}^{t} b^{(1) t, s} d s \quad \text { and } \quad \Lambda^{t}(b \circ \varphi(t)) .
$$

The first one is easily computed as $\partial_{z}^{2} Q(z)=-i, \partial_{\bar{z}}^{2} Q(z)=i$ and, with $c=b \circ \varphi(t)$,

$$
\begin{aligned}
-i\{c \circ \varphi(-s), Q(z)\}^{(2)}= & \left(\partial_{z}^{2}+\partial_{\bar{z}}^{2}\right)(c \circ \varphi(-s)) \\
= & {\left[\cosh (-2 s)\left(\partial_{z}^{2}+\partial_{\bar{z}}^{2}\right) c\right.} \\
& \left.+2 \sinh (-2 s) \partial_{\bar{z}} \partial_{z} c\right] \circ \varphi(-s)
\end{aligned}
$$


and thus

$$
\begin{aligned}
\int_{0}^{t} b^{(1) t, s} d s & =\int_{0}^{t}\left(\cosh (-2 s)\left(\partial_{z}^{2}+\partial_{\bar{z}}^{2}\right)+2 \sinh (-2 s) \partial_{\bar{z}} \partial_{z}\right) d s(b \circ \varphi(t)) \\
& =\left(\frac{1}{2} \sinh (2 t)\left(\partial_{z}^{2}+\partial_{\bar{z}}^{2}\right)+(1-\cosh (2 t)) \partial_{\bar{z}} \partial_{z}\right)(b \circ \varphi(t)) .
\end{aligned}
$$

Now we compute the second one. Since $L(t, 0) z=L^{*}(t, 0) z=z \cosh t$ and $A(t, 0) z=A^{*}(t, 0) z=$ $\bar{z} \sinh t$, we get $v_{t}=\cosh t \sinh t$ and then obtain directly

$$
\Lambda^{t}=(1-\cosh (2 t)) \partial_{\bar{z}} \partial_{z}+\frac{1}{2} \sinh (2 t)\left(\partial_{z}^{2}+\partial_{\bar{z}}^{2}\right)
$$

We thus obtain the same result with the two computations for the term of order 1 in $\varepsilon$.

Then we can show that

$$
\int_{\Delta_{t}^{k}} b^{(k) t, \bar{s}^{k}} d \bar{s}^{k}=\frac{1}{k !}\left(\Lambda^{t}\right)^{k}(b \circ \varphi(t))
$$

since

$$
\begin{aligned}
\int_{\Delta_{\mathrm{t}}^{\mathrm{k}}} \prod_{j=1}^{\mathrm{k}}\left(2 \sinh \left(-2 s_{j}\right) \partial_{\bar{z}} \partial_{z}+\cosh \left(-2 s_{j}\right)\left(\partial_{z}^{2}+\partial_{\bar{z}}^{2}\right)\right) \mathrm{d} \bar{s}^{k} & \\
& =\frac{1}{k !}\left((1-\cosh (-2 \mathrm{t})) \partial_{\bar{z}} \partial_{z}-\frac{1}{2} \sinh (-2 \mathrm{t})\left(\partial_{z}^{2}+\partial_{\bar{z}}^{2}\right)\right)^{k}
\end{aligned}
$$

because

$$
\frac{\mathrm{d}}{\mathrm{ds}}\left[(1-\cosh (-2 s)) \partial_{\bar{z}} \partial_{z}-\frac{1}{2} \sinh (-2 s)\left(\partial_{z}^{2}+\partial_{\bar{z}}^{2}\right)\right]=2 \sinh (-2 s) \partial_{\bar{z}} \partial_{z}+\cosh (-2 s)\left(\partial_{z}^{2}+\partial_{\bar{z}}^{2}\right) .
$$

Remark 5. Since these two formulae will be proven independently and the identification of each term of order $k$ in $\varepsilon$ in the expansion of the symbol is clear, we carry out a computation only on the formal level for the convenience of the reader to show the link between the two formulae in the general case.

We show (formally) that

Then it is simple to show that

$$
\frac{\mathrm{d}}{\mathrm{ds}} \Lambda^{\mathrm{s}}=\lambda^{\mathrm{s}}
$$

$$
\int_{\bar{s}^{k} \in \Delta_{t}^{k}} \lambda^{s_{k}} \lambda^{s_{k-1}} \cdots \lambda^{s_{1}} d \bar{s}^{k}=\frac{1}{k !}\left(\Lambda^{t}\right)^{k}
$$

as operators on $\mathcal{P}(\mathcal{Z})$ once the case $k=2$ is understood:

$$
\begin{aligned}
2 \int_{\overline{\mathrm{s}}^{2} \in \Delta_{\mathrm{t}}^{2}} \lambda^{s_{2}} \lambda^{s_{1}} \mathrm{~d} \bar{s}^{2} & =\int_{0}^{\mathrm{t}} \int_{0}^{s_{1}} \lambda^{s_{2}} \lambda^{s_{1}} \mathrm{~d} s_{2} \mathrm{~d} s_{1}+\int_{0}^{\mathrm{t}} \int_{0}^{s_{2}} \lambda^{s_{2}} \lambda^{s_{1}} \mathrm{~d} s_{1} \mathrm{ds} s_{2} \\
& =\int_{0}^{\mathrm{t}} \Lambda^{s_{1}} \lambda^{s_{1}} \mathrm{~d} s_{1}+\int_{0}^{\mathrm{t}} \lambda^{s_{2}} \Lambda^{s_{2}} \mathrm{~d} s_{2} \\
& =\left(\Lambda^{\mathrm{t}}\right)^{2} .
\end{aligned}
$$


In this computation we have used that $\Lambda^{0}=0$ as $A(0,0)=0$.

We first give $\lambda^{s}$ in a more explicit way. As $\partial_{\bar{z}}^{2} Q=i|\beta\rangle$ and $\partial_{z}^{2} Q=-i\langle\beta|$ we first get

$$
\lambda c=\left[\partial_{z}^{2}\left(c \circ \varphi^{-1}\right) \cdot|\beta\rangle+\langle\beta| . \partial_{\bar{z}}^{2}\left(c \circ \varphi^{-1}\right)\right] \circ \varphi
$$

with $\varphi=\varphi(t, 0)$ and omitting the time dependence everywhere. Then with $\varphi=\mathrm{L}+\mathrm{A}$ (and thus $\left.\varphi^{-1}=\mathrm{L}^{*}-\mathrm{A}^{*}\right)$ and $\left\langle z_{1}, A z_{2}\right\rangle=\left\langle z_{1} \otimes z_{2}, w_{A}\right\rangle$ we obtain

$$
\begin{aligned}
\lambda c(z)= & \partial_{z}^{2} \mathrm{c}(z) \cdot\left|\left(L^{* \vee 2}+A^{* \vee 2}\right) \beta\right\rangle+\left\langle\left(L^{* \vee 2}+A^{* \vee 2}\right) \beta\right| \cdot \partial_{\bar{z}}^{2} c(z) \\
& -2\left(\left\langle\left(I_{\mathcal{Z}} \otimes \partial_{\bar{z}} \partial_{z} c(z)^{*} L^{*}\right) \beta, w_{A}\right\rangle+\left\langle w_{A},\left(I_{\mathcal{Z}} \otimes \partial_{\bar{z}} \partial_{z} c(z) L^{*}\right) \beta\right\rangle\right) .
\end{aligned}
$$

Then we compute $\frac{d}{d s} \Lambda^{s}$ in several steps. The linear and antilinear parts of the equation $i \partial_{s} \varphi(s, 0) z=\partial_{\bar{z}} Q_{s}(\varphi(s, 0) z)$ give

$$
\begin{aligned}
\partial_{s} \mathrm{Lz} & =-i \alpha \mathrm{L} z+\left(\langle\mathrm{Az}| \vee \mathrm{I}_{\mathcal{Z}}\right)|\beta\rangle \\
\partial_{s} A z & =-i \alpha A z+\left(\langle\mathrm{Lz}| \vee \mathrm{I}_{\mathcal{Z}}\right)|\beta\rangle .
\end{aligned}
$$

We now show that $\partial_{s} v_{s}=\left|\left(L^{* \vee 2}+A^{* \vee 2}\right) \beta\right\rangle$,

$$
\begin{aligned}
\partial_{s}\left\langle z_{1} \otimes z_{2}, v_{s}\right\rangle= & \partial_{s}\left\langle\mathrm{~L} z_{1}, A z_{2}\right\rangle \\
= & \left\langle-i \alpha L z_{1}, A z_{2}\right\rangle+\left\langle\beta, A z_{2} \vee A z_{1}\right\rangle \\
& +\left\langle\mathrm{L} z_{1},-i \alpha A z_{2}\right\rangle+\left(\left\langle\mathrm{L} z_{2}\left|\vee\left\langle L z_{1}\right|\right) \mid \beta\right\rangle\right. \\
= & \left\langle\beta,(A \vee A)\left(z_{1} \vee z_{2}\right)\right\rangle+\left\langle(\mathrm{L} \vee \mathrm{L})\left(z_{1} \vee z_{2}\right), \beta\right\rangle \\
= & \left\langle z_{1} \vee z_{2},\left(\mathrm{~L}^{* V^{2}}+A^{* V^{2}}\right) \beta\right\rangle .
\end{aligned}
$$

And thus $\partial_{s}\left(\partial_{z}^{2} \cdot|v\rangle+\langle v| \cdot \partial_{\bar{z}}^{2}\right)=\partial_{z}^{2} \cdot\left|\left(L^{* \vee 2}+A^{* \vee 2}\right) \beta\right\rangle+\left\langle\left(L^{* \vee 2}+A^{* \vee 2}\right) \beta\right| . \partial_{\bar{z}}^{2}$.

We then show that

$$
\partial_{s} \operatorname{Tr}\left[A^{*} A \partial_{\bar{z}} \partial_{z} c(z)\right]=\left\langle\beta,\left(I_{\mathcal{Z}} \otimes L \partial_{\bar{z}} \partial_{z} c(z)\right) w_{A}\right\rangle+\left\langle w_{A},\left(I_{\mathcal{Z}} \otimes \partial_{\bar{z}} \partial_{z} c(z) L^{*}\right) \beta\right\rangle .
$$

We first observe that $\operatorname{Tr}\left[A^{*} A \partial_{\bar{z}} \partial_{z} c(z)\right]=\left\langle w_{A},\left(I_{\mathcal{Z}} \otimes \partial_{\bar{z}} \partial_{z} c(z)\right) w_{A}\right\rangle$. A simple calculation using $\partial_{s} A z=-i \alpha A z+\left(\langle L z| \vee I_{\mathcal{Z}}\right)|\beta\rangle$ shows that $\partial_{s} w_{A}=\left(-i \alpha \otimes I_{\mathcal{Z}}\right) w_{A}+\left(I_{\mathcal{Z}} \otimes L^{*}\right) \beta$ and this immediately gives the result.

\section{Classical evolution of a Wick polynomial under a quadratic evolution}

The adjoint of a $\mathbb{C}$-antilinear operator is defined in Appendix $\mathrm{A}$

Definition 4.1. A $\mathbb{C}$-antilinear operator $A$ on $\mathcal{Z}$ is said of Hilbert-Schmidt class if $\|A\|_{\mathcal{L}_{2}^{a}(\mathcal{Z})}:=$ $\left\|A A^{*}\right\|_{\mathcal{L}_{1}(\mathcal{Z})}^{1 / 2}$ is finite, where $\|\cdot\|_{\mathcal{L}_{1}(\mathcal{Z})}$ is the usual trace norm for $\mathbb{C}$-linear operators. The set of Hilbert-Schmidt antilinear operators is denoted by $\mathcal{L}_{2}^{\mathrm{a}}(\mathcal{Z})$. 
Let $\mathcal{X}(\mathcal{Z})=\mathcal{L}(\mathcal{Z})+\mathcal{L}_{2}^{\mathrm{a}}(\mathcal{Z})$ with norm

$$
\|\mathrm{T}\|_{\mathcal{X}(\mathcal{Z})}=\|\mathrm{L}\|_{\mathcal{L}(\mathcal{Z})}+\|\mathrm{A}\|_{\mathcal{L}_{2}^{a}(\mathcal{Z})}
$$

for $T=L+A$, where $L$ and $A$ are respectively $\mathbb{C}$-linear and $\mathbb{C}$-antilinear. The space $\mathcal{X}(\mathcal{Z})$ is a Banach algebra.

Remark 6. The norm $\|\mathrm{T}\|_{\mathcal{X}(\mathcal{Z})}$ is well defined as the decomposition $\mathrm{T}=\mathrm{L}+\mathrm{A}$ is unique $(\mathrm{L}=$ $\frac{1}{2}(\mathrm{~T}-i \mathrm{Ti})$ and $\left.A=\frac{1}{2}(\mathrm{~T}+i \mathrm{Ti})\right)$.

\subsection{Construction of the classical flow without the $\alpha$ term}

Let $\beta \in \mathcal{C}^{0}\left(\mathbb{R} ; \mathcal{Z}^{\vee 2}\right)$ and $Q_{\mathrm{t}}=\mathfrak{I}\left\langle\beta_{\mathrm{t}}, z^{\vee 2}\right\rangle$. Observe that $\partial_{\bar{z}} \mathrm{Q}(\mathrm{t})(z)=\mathfrak{i}\left(\mathrm{I}_{\mathcal{Z}} \vee\langle z|\right) \beta_{\mathrm{t}}$ and so $\left(\partial_{\bar{Z}} Q_{t}\right)_{t}$ is a continuous one parameter family of $\mathcal{X}(\mathcal{Z})$, so that the theory of ordinary differential equations in Banach algebras (see for example [21]) asserts that there exists a unique two parameters family $\varphi\left(t_{2}, t_{1}\right)$ of elements of $\mathcal{X}(\mathcal{Z})$ such that

$$
\left\{\begin{array}{rl}
i \partial_{\mathrm{t}} \varphi(\mathrm{t}, 0) & =\partial_{\bar{z}} \mathrm{Q}_{\mathrm{t}} \varphi(\mathrm{t}, 0) \\
\varphi(0,0) & =\mathrm{I}_{\mathcal{Z}}
\end{array},\right.
$$

with $\varphi$ of $\mathcal{C}^{1}$ class in both parameters such that for all $r, s$ and $t$,

$$
\varphi(t, s) \varphi(s, r)=\varphi(t, r)
$$

The classical flow $\varphi(t, s)$ is a symplectomorphism with respect to the symplectic form $\sigma\left(z_{1}, z_{2}\right)=$ $\mathfrak{I}\left\langle z_{1}, z_{2}\right\rangle$. It can be checked deriving

$$
\sigma\left(\varphi(t, s) z_{1}, \varphi(t, s) z_{2}\right)
$$

with respect to $t$.

\subsection{The strongly continuous dynamical system associated with $\left(\alpha_{t}\right)$}

We first state a proposition which is a direct consequence of Theorem X.70 in [27] in the unitary case. This proposition provides a set of assumptions ensuring the existence of a strongly continuous dynamical system associated with a family $\left(\alpha_{t}\right)_{t}$ of self-adjoint operators. Other more general situations can be considered as in 23, 24, for example.

Proposition 4.2. Let $\left(\alpha_{\mathfrak{t}}\right)_{t \in \mathbb{R}}$ be a family of self-adjoint operators on the Hilbert space $\mathcal{Z}$ satisfying the following conditions.

(1) The $\alpha_{t}$ have a common domain $\mathrm{D}$ (from which it follows by the closed graph theorem that $c(t, s)=\left(\alpha_{t}-\mathfrak{i}\right)\left(\alpha_{s}-\mathfrak{i}\right)^{-1}$ is bounded). 
(2) For each $z \in \mathcal{Z},(t-s)^{-1} c(t, s) z$ is uniformly strongly continuous and uniformly bounded in $s$ and $t$ for $t \neq s$ lying in any fixed compact interval.

(3) For each $z \in \mathcal{Z}, c(t) z=\lim _{s} \nearrow_{t}(t-s)^{-1} c(t, s) z$ exists uniformly for $t$ in each compact interval and $c(t)$ is bounded and strongly continuous in $t$.

The approximate propagator $\mathfrak{u}_{k}$ is defined by $\mathfrak{u}_{k}(t, s)=\exp \left(-(t-s) i \alpha_{\frac{j-1}{k}}\right)$ if $\frac{j-1}{k} \leq s \leq t \leq \frac{j}{k}$ and $u_{k}(t, r)=u_{k}(t, s) u_{k}(s, r)$.

Then for all $s, t$ in a compact interval and any $z \in \mathcal{Z}$,

$$
u(t, s) z=\lim _{k \rightarrow+\infty} u_{k}(t, s) z
$$

exists uniformly in $s$ and $t$. Further, if $z \in D$, then $u(t, s) z$ is in $D$ for all $s, t$ and satisfies

$$
\left\{\begin{aligned}
i \frac{\mathrm{d}}{\mathrm{dt}} \mathrm{u}(\mathrm{t}, \mathrm{s}) z & =\alpha_{\mathrm{t}} \mathrm{u}(\mathrm{t}, \mathrm{s}) z \\
\mathrm{u}(\mathrm{s}, \mathrm{s}) z & =z
\end{aligned}\right.
$$

\subsection{Construction of the classical flow with the $\alpha$ term}

Assume $\mathbf{H 1}$ and $\mathbf{H 2}$. Let $\hat{\varphi}$ be the solution of

$$
\left\{\begin{array}{rl}
i \partial_{\mathrm{t}} \hat{\varphi}(\mathrm{t}, 0) & =\partial_{\bar{z}} \hat{\mathrm{Q}}_{\mathrm{t}} \hat{\varphi}(\mathrm{t}, 0) \\
\hat{\varphi}(0,0) & =\mathrm{I}_{\mathcal{Z}}
\end{array},\right.
$$

with $\hat{Q}_{\mathrm{t}}(z)=\mathfrak{I}\left\langle\widehat{\beta}_{\mathrm{t}}, z^{\vee 2}\right\rangle, \widehat{\beta}_{\mathrm{t}}=\mathrm{u}_{\alpha}(\mathrm{t}, 0)^{* \vee 2} \beta_{\mathrm{t}}$. What we call here the solution of

$$
\left\{\begin{array}{rl}
i \partial_{t} \varphi(t, 0) & =\partial_{\bar{z}} Q_{t} \varphi(t, 0) \\
\varphi(0,0) & =I_{\mathcal{Z}}
\end{array},\right.
$$

with $\mathrm{Q}_{\mathrm{t}}=\left\langle z, \alpha_{\mathrm{t}} z\right\rangle+\mathfrak{I}\left\langle\beta_{\mathrm{t}}, z^{\vee 2}\right\rangle$ is

$$
\varphi(t, 0)=u_{\alpha}(t, 0) \circ \hat{\varphi}(t, 0)
$$

Depending on the assumptions on $\left(\alpha_{t}\right)$ it will be possible to precise if $\varphi$ solves Equation (4.1) in a usual sense (strongly, weakly, on some dense subset...).

With the particular set of assumptions of Theorem 4.2 we get that for all $z_{1} \in \mathrm{D}$ and $z_{2} \in \mathcal{Z}$,

$$
\left\{\begin{aligned}
i \partial_{t}\left\langle z_{1}, \varphi(t, 0) z_{2}\right\rangle & =\left\langle\alpha z_{1}, \varphi(t, 0) z_{2}\right\rangle+i\left\langle z_{1} \vee \varphi(t, 0) z_{2}, \beta\right\rangle \\
\varphi(0,0) & =I_{\mathcal{Z}}
\end{aligned}\right.
$$




\subsection{Composition of a Wick polynomial with the classical evolution}

The composition of a polynomial with the classical flow defines a time-dependent polynomial.

Definition 4.3. We define a norm on $\mathcal{P}(\mathcal{Z})$ by

$$
\|\mathrm{b}\|_{\mathcal{P}(\mathcal{Z})}=\sum_{\mathrm{p}, \mathrm{q}}\left\|\mathrm{b}_{\mathrm{p}, \mathrm{q}}\right\|_{\mathrm{q} \leftarrow \mathrm{p}}
$$

where $b=\sum_{p, q} b_{p, q}$ is a polynomial with $b_{p, q} \in \mathcal{P}_{p, q}(\mathcal{Z})$ and $\left\|b_{p, q}\right\|_{q \leftarrow p}$ is a shorthand for $\left\|\tilde{b}_{p, q}\right\|_{\mathcal{L}\left(\bigvee^{p} \mathcal{Z}, \bigvee^{\mathrm{q}} \mathcal{Z}\right)}$. For a polynomial $\mathrm{b}$ in $\mathcal{P}_{\mathrm{m}}(\mathcal{Z})$, we will sometimes write $\|\mathrm{b}\|_{\mathcal{P}_{\mathrm{m}}(\mathcal{Z})}$.

Proposition 4.4. Let $\mathrm{b} \in \mathcal{P}_{\mathrm{m}}(\mathcal{Z})$ be a polynomial, and $\varphi \in \mathcal{X}(\mathcal{Z})$. Then $\mathrm{b} \circ \varphi \in \mathcal{P}_{\mathrm{m}}(\mathcal{Z})$ and we have the estimate

$$
\|\mathrm{b} \circ \varphi\|_{\mathcal{P}_{\mathrm{m}}(\mathcal{Z})} \leq\|\varphi\|_{\mathcal{X}(\mathcal{Z})}^{\mathrm{m}}\|\mathrm{b}\|_{\mathcal{P}_{\mathrm{m}}(\mathcal{Z})}
$$

Proof. The proof is essentially the same as in Proposition 2.12 of [1].

\section{Quantum evolution of a Wick polynomial}

\subsection{Without the $\alpha$ term}

Definition 5.1. Let $\beta \in \mathcal{C}^{0}\left(\mathbb{R} ; \mathcal{Z}^{\vee 2}\right)$ and $\mathrm{Q}_{\mathrm{t}}(z)=\mathfrak{I}\left\langle\beta_{\mathrm{t}}, z^{\vee 2}\right\rangle$. A family $\mathrm{U}(\mathrm{t}, \mathrm{s})$ of unitary operators on $\mathcal{H}$ defined for $s, t$ real is a solution of

$$
\left\{\begin{aligned}
i \partial_{t} U(t, 0) & =\frac{Q_{t}^{W i c k}}{\varepsilon} U(t, 0) \\
U(0,0) & =I_{\mathcal{H}}
\end{aligned}\right.
$$

if

(1) $\mathrm{U}(\mathrm{t}, \mathrm{s})$ is strongly continuous in $\mathcal{H}$ with respect to $\mathrm{s}$, $\mathrm{t}$ with $\mathrm{U}(\mathrm{s}, \mathrm{s})=\mathrm{I}$,

(2) $\mathrm{U}(\mathrm{t}, \mathrm{r})=\mathrm{U}(\mathrm{t}, \mathrm{s}) \mathrm{U}(\mathrm{s}, \mathrm{r}), \mathrm{r} \leq \mathrm{s} \leq \mathrm{t}$,

(3) $i \frac{d}{d t} U(t, s) y$ exists for almost every $t$ (depending on $s$ ) and is equal to $Q_{t}^{W i c k} U(t, s) y$,

(4) $i \varepsilon \frac{d}{d s} U(t, s) y=-U(t, s) Q_{s}^{W i c k} y, y \in \mathcal{D}(N+1), 0 \leq s \leq t$.

This definition is made to fit the general framework of Theorems 4.1 and 5.1 of [23]. More precisely we may check the following theorem.

Theorem 5.2. Let $\beta \in \mathcal{C}^{0}\left(\mathbb{R} ; \mathcal{Z}^{\vee 2}\right)$ and $\mathrm{Q}_{\mathrm{t}}(z)=\mathfrak{I}\left\langle\beta_{\mathrm{t}}, z^{\vee 2}\right\rangle$.

Then the quantum flow equation (5.1) associated to the family $\frac{1}{\varepsilon} \mathrm{Q}_{\mathrm{t}}$ has a unique solution. This solution preserves the sets $\mathcal{D}\left(\langle\mathrm{N}\rangle^{\mathrm{k} / 2}\right)$ for $\mathrm{k} \geq 2$.

To establish this theorem we will use the following estimates. 
Lemma 5.1. Let $\beta \in \mathcal{Z}^{\vee 2}$ and $\mathrm{Q}(z)=\mathfrak{I}\left\langle\beta, z^{\vee 2}\right\rangle$. Then, on $\mathcal{H}_{\text {fin }}$, and for $\mathrm{k} \geq 1$, $\mathrm{Q}^{\text {Wick }}$ satisfies the estimates

$$
\left\|\mathrm{Q}^{W i c k} / \varepsilon \Psi\right\| \leq \frac{3}{2}\|\beta\|_{\mathcal{Z}^{\vee 2}}\|(\mathrm{~N} / \varepsilon+1) \Psi\|
$$

and

$$
\pm i\left[\mathrm{Q}^{W i c k} / \varepsilon,(\mathrm{N} / \varepsilon+1)^{\mathrm{k}}\right] \leq 3^{\mathrm{k}} \sqrt{2}\|\beta\|_{\mathcal{Z}^{\vee 2}}(\mathrm{~N} / \varepsilon+1)^{\mathrm{k}}
$$

The second estimate is in the sense of quadratic forms, for all $\Psi \in \mathcal{H}_{\text {fin }}$,

$$
\begin{aligned}
\pm i\left(\left\langle\frac{1}{\varepsilon} Q^{W i c k} \Psi,(N / \varepsilon+1)^{k} \Psi\right\rangle\right. & \left.-\left\langle(N / \varepsilon+1)^{k} \Psi, \frac{1}{\varepsilon} Q^{W i c k} \Psi\right\rangle\right) \\
& \leq \frac{3^{k}}{\sqrt{2}}\|\beta\|_{\mathcal{Z}^{\vee 2}}\left\langle\Psi,(N / \varepsilon+1)^{k} \Psi\right\rangle .
\end{aligned}
$$

Proof. The first estimate is a consequence of $n+2 \leq 2(n+1)$ associated to

$$
\left.\frac{2 i}{\mathcal{E}} Q^{W i c k}\right|_{\mathcal{Z}^{\vee n}}=\sqrt{n(n-1)}\left\langle\beta\left|\vee I_{\bigvee^{n-2} \mathcal{Z}}-\sqrt{(n+2)(n+1)}\right| \beta\right\rangle \vee I_{\bigvee^{n} \mathcal{Z}}
$$

For the second estimate, consider $\frac{2 i}{\varepsilon}\left\langle\Psi,\left[(1+N / \varepsilon)^{k}, Q^{W i c k}\right] \Psi\right\rangle$. The first term of this commutator is

$$
\sum_{n}(n+1)^{k}\left(\sqrt{(n+2)(n+1)}\left\langle\Psi^{(n)} \vee\left\langle\beta \mid, \Psi^{(n+2)}\right\rangle-\sqrt{n(n-1)}\left\langle\Psi^{(n)}, \mid \beta\right\rangle \vee \Psi^{(n-2)}\right\rangle\right) .
$$

Then we deduce easily the second term and a reindexation gives the following form for the whole commutator:

$$
\begin{aligned}
\sum_{n}\left[(n+1)^{k}-((n+2)+1)^{k}\right] \sqrt{(n+2)(n+1)} & \\
& \times\left(\left\langle\Psi^{(n)} \vee\left\langle\beta \mid, \Psi^{(n+2)}\right\rangle+\left\langle\Psi^{(n+2)}, \mid \beta\right\rangle \vee \Psi^{(n)}\right\rangle\right) .
\end{aligned}
$$

Newton's binomial formula and the inequalities $\sum_{l=0}^{k-1}\left(\begin{array}{l}k \\ l\end{array}\right) 2^{k-l} \leq 3^{k}$ and $(n+1)^{l} \leq(n+1)^{k-1}$ yield

$$
(n+1)^{k}-((n+2)+1)^{k} \leq 3^{k}(n+1)^{k-1} .
$$

Using also $n+2 \leq 2(n+1)$ to control $\sqrt{(n+2)(n+1)}$ we obtain

$$
\pm i\left\langle\Psi,\left[(1+N / \varepsilon)^{k}, \frac{Q^{W i c k}}{\varepsilon}\right] \Psi\right\rangle \leq \frac{1}{2} \sum_{n} 3^{k}(n+1)^{k-1} \sqrt{2}(n+1)\left\|\Psi^{(n)}\right\|\|\beta\|_{\mathcal{Z}^{\vee 2}}\left\|\Psi^{(n+2)}\right\| .
$$

Cauchy-Schwarz's inequality gives the claimed estimate.

Lemma 5.2. Let $\beta \in \mathcal{Z}^{\vee 2}$ and $\mathrm{Q}(z)=\mathfrak{I}\left\langle\beta, z^{\vee 2}\right\rangle$. Then $\mathrm{Q}^{\text {Wick }}$ is essentially self-adjoint on $\mathcal{H}_{\text {fin }}$ and its closure is essentially self-adjoint on any other core for $\mathrm{N} / \varepsilon+1$. Inequalities (5.2) and (5.3) still hold on $\mathcal{D}(\mathrm{N} / \varepsilon+1)$. 
We still denote by $\mathrm{Q}^{\text {Wick }}$ this self-adjoint extension.

Proof. We apply the commutators Theorem X.37 of [27] with the estimates of Lemma 5.1 for $k=$ 1.

Lemma 5.3. If a solution of the quantum flow equation (5.1) exists then it leaves $\mathcal{Q}\left((\mathrm{N} / \varepsilon+1)^{\mathrm{k}}\right)=$ $\mathcal{D}\left((\mathrm{N} / \varepsilon+1)^{\mathrm{k} / 2}\right)$ invariant for any integer $\mathrm{k} \geq 2$.

In the time-independent case the estimate

$$
\|\mathrm{U}(\mathrm{t}, 0)\|_{\mathcal{L}\left(\mathcal{D}\left((\mathrm{N} / \varepsilon+1)^{\mathrm{k} / 2}\right)\right)} \leq \exp \left(3^{\mathrm{k}} \sqrt{2}\|\beta\||\mathrm{t}|\right)
$$

holds.

Proof. From Lemma 5.2, for any $\mathrm{k} \geq 2, \mathcal{D}\left((\mathrm{N} / \varepsilon+1)^{\mathrm{k} / 2}\right) \subset \mathcal{D}\left(\mathrm{Q}^{\text {Wick }}\right)$. We can adapt the proof of Theorem 2 of [10] to the case of the quantization of a continuous one parameter family of quadratic polynomials with the estimates of Lemma 5.1 .

Proof of theorem 5.2. We use Theorems 4.1 and 5.1 of 23 with the family of operators $i Q(t){ }^{\text {Wick }} / \varepsilon$ (here we directly consider the self-adjoint extension of $\left.\mathrm{Q}_{t}^{\text {Wick }} / \varepsilon\right)$. We set $\mathrm{Y}=\mathcal{D}\left((\mathrm{N} / \varepsilon+1)^{\mathrm{k} / 2}\right)$.

(1) This family is stable in the sense that $\left\|\prod_{j=1}^{k} e^{-i s_{j} Q\left(t_{j}\right)^{\text {Wick }} / \varepsilon}\right\|_{\mathcal{L}(\mathcal{H})} \leq 1$ (we actually have an equality here).

(2) The space $Y$ is admissible for this family in the sense that for each $t$, $\left(i Q_{t}^{\text {Wick }} / \varepsilon+\lambda\right)^{-1}$ leaves $\mathrm{Y}$ invariant and

$$
\left\|\left(i Q_{t}^{W i c k} / \varepsilon+\lambda\right)^{-1}\right\|_{\mathcal{L}(Y)} \leq\left(\lambda-3^{k} \sqrt{2}\|\beta\|\right)^{-1}
$$

for $\Re \lambda>3^{k} \sqrt{2}\|\beta\|$.

This is true because, as we have seen in Lemma 5.3. $\left(e^{-i s Q_{t}^{\text {Wick }} / \varepsilon}\right)_{s \in \mathbb{R}}$ leaves $Y$ invariant and, thanks to the estimate of the same lemma, we can apply the resolvent formula

$$
\left(i Q_{t}^{\text {Wick }} / \varepsilon+\lambda\right)^{-1}=\int_{0}^{+\infty} e^{-\lambda s} e^{-i s Q_{t}^{W i c k} / \varepsilon} d s
$$

and obtain the desired estimate.

(3) $\mathrm{Y} \subset \mathcal{D}\left(\mathrm{Q}_{\mathrm{t}}^{\text {Wick }} / \varepsilon\right)$ so that $\mathrm{Q}_{\mathrm{t}}^{\text {Wick }} / \varepsilon \in \mathcal{L}(\mathrm{Y}, \mathcal{H})$ for each $\mathrm{t}$, and the map $\mathrm{t} \rightarrow \mathrm{Q}_{\mathrm{t}}^{\text {Wick }} / \varepsilon \in$ $\mathcal{L}(\mathrm{Y}, \mathcal{H})$ is continuous.

(4) $\mathrm{Y}=\mathcal{D}\left((\mathrm{N} / \varepsilon+1)^{\mathrm{k} / 2}\right)$ is reflexive.

Theorems 4.1 and 5.1 of 23 thus apply and give the existence of an evolution operator.

The preservation of the set $\mathcal{D}\left((\mathrm{N} / \varepsilon+1)^{\mathrm{k} / 2}\right)$ comes from the application of Lemma 5.3 to the solution of the time-dependent problem. To conclude it is then enough to observe that the domains $\mathcal{D}\left(\langle\mathrm{N}\rangle^{\mathrm{k} / 2}\right)$ and $\mathcal{D}\left((\mathrm{N} / \varepsilon+1)^{\mathrm{k} / 2}\right)$ are the same and have equivalent norms. 


\subsection{With the $\alpha$ term}

Assume $\mathbf{H 1}$ and $\mathbf{H 2}$. Let $\mathfrak{u}$ be the solution of

$$
\left\{\begin{aligned}
i \partial_{t} \widehat{U}(t, 0) & =\frac{\hat{Q}_{t}^{\text {Wick }}}{\varepsilon} \widehat{U}(t, 0) \\
\widehat{U}(0,0) & =I_{\mathcal{H}}
\end{aligned}\right.
$$

with $\hat{Q}_{\mathrm{t}}(z)=\mathfrak{I}\left\langle\hat{\beta}_{\mathrm{t}}, z^{\vee 2}\right\rangle, \hat{\beta}_{\mathrm{t}}=\mathfrak{u}_{\alpha}(\mathrm{t}, 0)^{* \vee 2} \beta_{\mathrm{t}}$. What we call here the solution of

$$
\left\{\begin{aligned}
i \partial_{t} U(t, 0) & =\frac{Q_{t}^{\text {Wick }}}{\varepsilon} U(t, 0) \\
U(0,0) & =I_{\mathcal{H}}
\end{aligned}\right.
$$

with $\mathrm{Q}_{\mathrm{t}}=\left\langle z, \alpha_{\mathrm{t}} z\right\rangle+\mathfrak{I}\left\langle\beta_{\mathrm{t}}, z^{\vee 2}\right\rangle$ is

$$
\mathrm{u}(\mathrm{t}, 0)=\Gamma\left(\mathrm{u}_{\alpha}(\mathrm{t}, 0)\right) \circ \hat{\mathrm{u}}(\mathrm{t}, 0) .
$$

\section{Removal of the $\alpha$ part}

Proposition 6.1. Assume $\mathbf{H 1}$ and H2. Suppose Theorems 3.1 and 3.2 hold with a null one parameter family of self-adjoint operators on $\mathcal{Z}$, and $\hat{\beta}_{t}=u_{\alpha}(t, 0)^{* \vee 2} \beta_{t}$. We denote with a hat the quantities associated with this solution. Then Theorems 3.1 and 3.2 hold.

Proof. For Equation 3.3 we forget during the proof the $(t, 0)$ dependency in our notations and write

$$
\int_{\Delta_{\mathrm{t}}^{0}} \mathrm{~b}^{(0) t, \bar{s}^{0}} d \bar{s}^{0}
$$

instead of $b^{(0), t}$. Then

$$
\begin{aligned}
\mathrm{u}^{*} \mathrm{~b}^{\text {Wick }} \mathrm{u} & =\hat{\mathrm{u}}^{*} \Gamma\left(\mathrm{u}_{\alpha}^{*}\right) \mathrm{b}^{\text {Wick }} \Gamma(\mathrm{u}) \hat{\mathrm{u}} \\
& =\hat{\mathrm{u}}^{*}\left(\mathrm{~b} \circ \mathrm{u}_{\alpha}\right)^{\text {Wick }} \hat{\mathrm{u}} \\
& =\sum_{\mathrm{k}=0}^{\left\lfloor\frac{\mathrm{m}}{2}\right\rfloor}\left(\frac{\varepsilon}{2}\right)^{k} \int_{\Delta_{\mathrm{t}}^{k}}\left({\widehat{\mathrm{b} \circ \mathrm{u}_{\alpha}}}^{(k) t, \overline{\mathrm{s}}^{k}}\right)^{W i c k} \mathrm{~d} \overline{\mathrm{s}}^{\mathrm{k}}
\end{aligned}
$$

where the $\hat{b}^{(k) t, \bar{s}^{k}}$ are defined recursively by

$$
\left\{\begin{aligned}
\hat{\mathrm{b}}^{(0) \mathrm{t}}(z) & =\mathrm{b} \circ \hat{\varphi} \\
\hat{\mathrm{b}}^{(k+1) t}, \overline{\mathrm{s}}^{k+1} & =\hat{\lambda}^{s_{k+1}} \hat{\mathrm{b}}^{(k) t, \bar{s}^{k}}
\end{aligned}\right.
$$

with $\hat{\lambda}^{s} c=-i\left\{c \circ \hat{\varphi}(0, s), \hat{Q}_{s}\right\}^{(2)} \circ \hat{\varphi}(s, 0)$ for any polynomial $c$. Thus it suffices to prove that

$$
{\widehat{\mathrm{b} \circ \mathrm{u}_{\alpha}}}^{(k) t, \overline{\mathrm{s}}^{\mathrm{k}}}=\mathrm{b}^{(\mathrm{k}) \mathrm{t}, \overline{\mathrm{s}}^{\mathrm{s}}} \text {. }
$$


This is clear for $k=0$ as $\mathfrak{u}_{\alpha} \circ \hat{\varphi}=\varphi$. Then we observe that

$$
\begin{aligned}
\hat{\lambda}^{s} \mathrm{c} & =-i\left\{c \circ \hat{\varphi}^{-1}, \hat{\mathrm{Q}}\right\}^{(2)} \circ \hat{\varphi} \\
& =-i\left\{\mathrm{c} \circ \varphi^{-1} \circ \mathrm{u}_{\alpha}, \hat{\mathrm{Q}}\right\}^{(2)} \circ \mathrm{u}_{\alpha}^{-1} \circ \varphi \\
& =-i\left\{\mathrm{c} \circ \varphi^{-1}, \mathrm{Q}\right\}^{(2)} \circ \varphi
\end{aligned}
$$

where we used that $\partial_{z}^{2}\langle z, \alpha z\rangle=0, \partial_{\bar{z}}^{2}\langle z, \alpha z\rangle=0$ and $\beta_{t}=u_{\alpha}(t, 0)^{\vee 2} \hat{\beta}_{t}$.

We can thus restrict our proof to the case of a polynomial $Q_{t}$ of the form $Q_{t}(z)=\mathfrak{I}\left\langle\beta_{t}, z^{\vee 2}\right\rangle$ with $\beta_{\mathrm{t}} \in \mathcal{C}^{0}\left(\mathbb{R} ; \mathcal{Z}^{\vee 2}\right)$ and no $\left(\alpha_{\mathrm{t}}\right)$ term.

\section{A Dyson type expansion formula for the Wick symbol of the evolved quantum observable}

In this section we prove Theorem 3.1.

Proof. We first prove that the formula, for $\mathrm{c} \in \mathcal{P}_{\leq \mathrm{m}}(\mathcal{Z})$,

$$
u(0, s)(c \circ \varphi(0, s))^{\text {Wick }} u(s, 0)=c^{\text {Wick }}-\frac{i \varepsilon}{2} \int_{0}^{s} u(0, \sigma)\left\{c \circ \varphi(0, \sigma), Q_{\sigma}\right\}^{(2) \text { Wick }} u(\sigma, 0) d \sigma
$$

holds as an equality of continuous operators from $\mathcal{D}\left(\langle\mathrm{N}\rangle^{\mathrm{m} / 2}\right)$ to $\mathcal{H}$, with $\langle\mathrm{N}\rangle=\left(\mathrm{N}^{2}+1\right)^{1 / 2}$. This is a consequence of the fact that the derivative of the left hand term as a function of $s$ is $-\frac{i \varepsilon}{2} \mathrm{U}(0, s)\left\{\mathrm{c} \circ \varphi(0, s), \mathrm{Q}_{s}\right\}^{(2) \text { Wick }} \mathrm{U}(\mathrm{s}, 0)$ as it can be seen from the relation

$$
i \partial_{\sigma}(c \circ \varphi(0, \sigma))=-\partial_{z}(c \circ \varphi(0, \sigma)) \cdot \partial_{\bar{z}} Q_{\sigma}+\partial_{z} Q_{\sigma} \cdot \partial_{\bar{z}}(c \circ \varphi(0, \sigma))
$$

and Proposition 2.1. Applying the previous formula with $c=b^{(K) t, \bar{s}^{K}}$ we get recursively

$$
\begin{aligned}
& u(0, t) b^{\text {Wick }} u(t, 0) \\
& =\sum_{k=0}^{K-1}\left(\frac{\varepsilon}{2}\right)^{k} \int_{\bar{s}^{k} \in \Delta_{t}^{k}}\left(b^{(k) t, \bar{s}^{k}}\right)^{W i c k} d \bar{s}^{k} \\
& +\left(\frac{\varepsilon}{2}\right)^{K} \int_{\bar{s}^{K} \in \Delta_{t}^{K}} \mathrm{U}\left(0, s_{K}\right)\left(b^{(K) t, \bar{s}^{K}} \circ \varphi\left(0, s_{K}\right)\right)^{\text {Wick }} \mathrm{u}\left(s_{K}, 0\right) d \bar{s}^{K} .
\end{aligned}
$$

This process gives a null remainder as soon as $K>m / 2$ as for $K \leq\lfloor m / 2\rfloor$, since the polynomial $b^{(K) \bar{s}^{K}}$ is of total order $m-2 K$.

\section{An exponential type expansion formula for the Wick sym- bol of the evolved observable}

In this section we prove Theorem 3.2 . 


\subsection{Quantum evolution as a Bogoliubov implementation}

Some basic facts about symplectomorphisms are recalled in Appendix A

Definition 8.1. A symplectomorphism $\mathrm{T}$ is called implementable if and only if there exists a unitary operator $\mathrm{U}$ on $\mathcal{H}$, called a Bogoliubov implementer of $\mathrm{T}$, such that

$$
\forall \xi \in \mathcal{Z}, \mathrm{U}^{*} \mathrm{~W}(\xi) \mathrm{U}=\mathrm{W}(\mathrm{T} \xi) .
$$

Proposition 8.2. Assume $\alpha_{\mathrm{t}} \equiv 0$ and H2. Let $\mathrm{Q}_{\mathrm{t}}=\mathfrak{I}\left\langle\beta_{\mathrm{t}}, z^{\vee 2}\right\rangle, \varphi(\mathrm{t}, \mathrm{s})$ the associated classical evolution (see Section 4) and $U(t, s)$ the associated quantum evolution (see Section 5). Then for all $t$ in $\mathbb{R}, U(t, 0)$ is a Bogoliubov implementer of $-i \varphi(0, t) i$.

Remark 7 . Note that the symplectomorphism $\varphi(0, t)$ is only $\mathbb{R}$-linear and not $\mathbb{C}$-linear in general, and thus $-i \varphi(0, t) i \neq \varphi(0, t)$.

Proof. We begin with a formal computation which will be justified further. It suffices to show that

$$
i \varepsilon \partial_{t}[U(0, t) W(-i \varphi(t, 0) i \xi) U(t, 0)]=0 .
$$

Computing this derivative and omitting the time and $-i \varphi(t, 0) i \xi$ dependencies in our notations, we get with $\mathrm{U}(\mathrm{t}, 0)=\mathrm{U}$

$$
\mathrm{U}^{*} \mathrm{~W}\left\{-\mathrm{W}^{*} \mathrm{Q}^{\text {Wick }} \mathrm{W}+\mathrm{Q}^{\text {Wick }}+\mathrm{W}^{*} i \varepsilon \partial_{\mathrm{t}} \mathrm{W}\right\} \mathrm{U} .
$$

Then from Proposition 2.10 (iii) in [1, the differential formula of Weyl operators recalled in Proposition 8.3 below and with $f_{t}=-i \varphi(t, 0) i \xi$ it suffices to show that

$$
\mathrm{Q}\left(z+\frac{i \varepsilon}{\sqrt{2}} f_{t}\right)=Q(z)+i \varepsilon\left(\frac{i \varepsilon}{2} \mathfrak{J}\left\langle f_{t}, \partial_{t} f_{t}\right\rangle+i \sqrt{2} \mathfrak{R}\left\langle\partial_{t} f_{t}, z\right\rangle\right)
$$

to get the result. This equality results from the expansion of $\mathrm{Q}(z)=\mathfrak{I}\left\langle\beta, z^{\vee 2}\right\rangle$, recalling that $i \partial_{t} \varphi(t, 0) \xi=\partial_{\bar{z}} \mathrm{Q}(\varphi(t, 0) \xi)$, and observing that $\partial_{\bar{z}} \mathrm{Q}(z)=\mathfrak{i}\left(\langle z| \vee \mathrm{I}_{\mathcal{Z}}\right)|\beta\rangle$. We now need to clarify the meaning of this computation. It suffices to show that the quantity

$$
\langle\Phi, \mathrm{U}(0, \mathrm{t}) \mathrm{W}(-\mathrm{i} \varphi(t, 0) i \xi) \mathrm{U}(\mathrm{t}, 0) \Psi\rangle
$$

is constant for $\Psi, \Phi$ in $\mathcal{D}(N+1)$. Since this domain is preserved by the operators $U(t, s)$, the Weyl operators are weakly derivable on this domain (see next proposition), and $\mathrm{U}(\mathrm{t}, \mathrm{s})$ is derivable on this domain, then we get the justification of the previous formal computation.

Proposition 8.3. Let $z, h$ be vectors in $\mathcal{Z}, \mathrm{t}$ be a real parameter and $\varphi, \psi$ be in the domain of $\Phi(\mathrm{h})$. Then

$$
\begin{aligned}
\lim _{\mathrm{t} \rightarrow 0} \frac{1}{\mathrm{t}}(\langle\varphi,[\mathrm{W}(z+\mathrm{th})-W(z)] \psi\rangle) & =\left\langle\varphi, W(z)\left[i \Phi(h)+\frac{i \varepsilon}{2} \mathfrak{I}\langle z, h\rangle+\right] \psi\right\rangle \\
& =\left\langle\varphi,\left[i \Phi(h)-\frac{\mathfrak{i} \varepsilon}{2} \mathfrak{I}\langle z, h\rangle\right] W(z) \psi\right\rangle .
\end{aligned}
$$


Proof. For the first equality. The Weyl commutation relations give

$$
\begin{aligned}
& \frac{1}{\mathrm{t}}\langle\varphi,[\mathrm{W}(z+\mathrm{th})-\mathrm{W}(z)] \psi\rangle=\frac{1}{\mathrm{t}}\left\langle\mathrm{W}(-z) \varphi,\left[e^{\frac{\mathrm{i} \varepsilon}{2} \mathfrak{J}\langle z, \text { th }\rangle} \mathrm{W}(\mathrm{th})-\mathrm{I}_{\mathcal{Z}}\right] \psi\right\rangle
\end{aligned}
$$

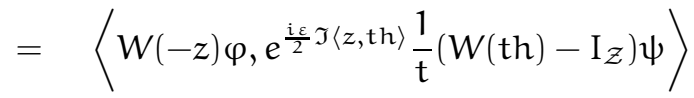

$$
\begin{aligned}
& +\frac{1}{\mathrm{t}}\left(e^{\frac{i \varepsilon}{2} \mathfrak{I}\langle z, \mathrm{th}\rangle}-1\right)\langle\mathrm{W}(-z) \varphi, \psi\rangle \\
& \underset{\mathrm{t} \rightarrow 0}{\rightarrow}\left\langle\varphi, W(z)\left[i \Phi(h)+\frac{i \varepsilon}{2} \mathfrak{I}\langle z, h\rangle\right] \psi\right\rangle \text {. }
\end{aligned}
$$

The convergence of the first term is due to the continuous one parameter group structure of $W(t h)$. The other equality is obtained in the same way.

\subsection{Action of Bogoliubov transformations on Wick symbols}

A theorem due to Shale (see [29]) characterizes implementable symplectomorphisms. We quote here a version of this theorem fitting our needs.

Theorem 8.4 (Shale, 1962). A symplectomorphism $\mathrm{T}$ is implementable if and only if the $\mathbb{C}$-linear part of $\mathrm{T}^{*} \mathrm{~T}-\mathrm{Id}$ is trace class.

We can now quote the main result of this part.

Theorem 8.5. Let $\mathrm{T}=\mathrm{L}+\mathrm{A}$ with $\mathrm{L} \mathbb{C}$-linear and $\mathrm{A} \mathbb{C}$-antilinear, be an implementable symplectomorphism with a Bogoliubov implementer $\mathrm{U}$ preserving $\mathcal{D}\left(\langle\mathrm{N}\rangle^{\mathrm{k} / 2}\right)$ for any integer $\mathrm{k} \geq 2$, then for any polynomial $\mathrm{b}$ in $\mathcal{P}_{\leq \mathrm{m}}(\mathcal{Z})$ with $\mathrm{m} \geq 2$,

$$
\mathrm{U}^{*} \mathrm{~b}^{\text {Wick }} \mathrm{U}=\left(\mathrm{e}^{\frac{\varepsilon}{2} \wedge[\mathrm{T}]}\left[\mathrm{b}\left(\mathrm{T}^{*} \cdot\right)\right]\right)^{\text {Wick }}
$$

as an equality of continuous operators from $\mathcal{D}\left(\langle\mathrm{N}\rangle^{\mathrm{m} / 2}\right)$ to $\mathcal{H}$, with $\langle\mathrm{N}\rangle=\left(\mathrm{N}^{2}+1\right)^{1 / 2}$, where

- the exponential is a finite expansion whose rank depends on the degree of the polynomial $\mathbf{b}$,

- the operator $\Lambda[\mathrm{T}]$ is defined on any polynomial $c$ by

$$
\Lambda[T] c(z)=\operatorname{Tr}\left[-2 A A^{*} \partial_{\bar{z}} \partial_{z} c(z)\right]+\left\langle v\left|\cdot \partial_{\bar{z}}^{2} \mathrm{c}(z)+\partial_{z}^{2} \mathrm{c}(z) \cdot\right| v\right\rangle
$$

with $v \in \otimes^{2} \mathcal{Z}$ the vector such that for all $z_{1}, z_{2} \in \mathcal{Z},\left\langle z_{1} \otimes z_{2}, v\right\rangle=\left\langle z_{1}, \mathrm{LA}^{*} z_{2}\right\rangle$.

In order to prove this result, we use intermediate steps.

(1) We prove that $\mathbf{U}^{*} \mathbf{b}^{W^{W e y}} \mathrm{l} U=\mathbf{b}\left(\mathbf{T}^{*} .\right)^{\text {Weyl }}$ in finite dimension. 
(2) We use the Fourier transform and the formula

$$
\mathrm{b}^{\text {Weyl }}=\frac{1}{(\pi \varepsilon / 2)^{\mathrm{d}}}\left(\mathrm{b} * \mathrm{e}^{-\frac{|z|^{2}}{\varepsilon / 2}}\right)^{\text {Wick }}
$$

to get the result in finite dimension.

(3) We extend the result to infinite dimension.

\subsection{Action of Bogoliubov transformations on Weyl quantizations of poly- nomials in finite dimension}

Definition 8.6. In a finite-dimensional Hilbert space $\mathcal{Z}$ identified with $\mathbb{C}^{\mathrm{d}}$, the symplectic Fourier transform is defined by

$$
\mathcal{F}^{\sigma}[\mathrm{f}](z)=\int_{\mathcal{Z}} e^{2 \pi i \sigma\left(z, z^{\prime}\right)} \mathrm{f}\left(z^{\prime}\right) \mathrm{L}\left(\mathrm{d} z^{\prime}\right)
$$

where $L$ denotes the Lebesgue measure, and $f$ is any Schwartz tempered distribution. We associate with each polynomial $\mathbf{b} \in \mathcal{P}_{\mathbf{p}, \mathfrak{q}}(\mathcal{Z})$ a Weyl observable by

$$
\mathrm{b}^{\text {Weyl }}=\int_{\mathcal{Z}} \mathcal{F}^{\sigma}[\mathrm{b}](z) W(-\mathfrak{i} \sqrt{2} \pi z) L(\mathrm{~d} z) .
$$

This formula has a meaning as an equality of quadratic forms on $\mathcal{S}(\mathcal{Z})$ since for any $\Phi, \Psi$ in $\mathcal{S}(\mathcal{Z})$, $z \mapsto\langle\Phi, W(-i \sqrt{2} \pi z) \Psi\rangle$ and its derivative are continuous bounded functions and $\mathcal{F}^{\sigma}[\mathrm{b}]$ is made of derivatives of the delta function.

Proposition 8.7. Let $\mathrm{b} \in \mathcal{P}_{\leq \mathrm{m}}(\mathcal{Z})$ with $\mathrm{m} \geq 2$ be a polynomial on a finite-dimensional Hilbert space $\mathcal{Z}$. Let $\mathrm{T}$ be an implementable symplectomorphism with implementation $\mathrm{U}$ preserving the domain $\mathcal{D}\left(\langle\mathrm{N}\rangle^{\mathrm{m} / 2}\right)$. Then

$$
\mathrm{U}^{*} \mathrm{~b}^{\text {Weyl }} \mathrm{u}=\mathrm{b}\left(\mathrm{T}^{*} \cdot\right)^{\text {Weyl }}
$$

as a continuous operator from $\mathcal{D}\left(\langle\mathrm{N}\rangle^{\mathrm{m} / 2}\right)$ to $\mathcal{H}$.

Proof. We compute, in the sense of quadratic forms on $\mathcal{S}(\mathcal{Z})$,

$$
\begin{aligned}
\mathrm{U}^{*} \mathrm{~b}^{\text {Weyl } \mathrm{u}} & =\int \mathcal{F}^{\sigma}[\mathrm{b}](z) \mathrm{W}(-\sqrt{2} \pi \mathrm{Tiz}) \mathrm{L}(\mathrm{d} z) \\
& =\int \mathcal{F}^{\sigma}[\mathrm{b}]\left(\mathrm{T}^{*} z\right) W(-\mathrm{i} \sqrt{2} \pi z) \mathrm{L}(\mathrm{d} z) \\
& =\int \mathcal{F}^{\sigma}\left[\mathrm{b}\left(\mathrm{T}^{*} \cdot\right)\right](z) W(-\mathrm{i} \sqrt{2} \pi z) \mathrm{L}(\mathrm{d} z) \\
& =\mathrm{b}\left(\mathrm{T}^{*} \cdot\right)^{\text {Weyl }}
\end{aligned}
$$

where we made use of the relation $T i=i\left(T^{*}\right)^{-1}$, the volume preservation of $\mathrm{T}^{*}$ in $\mathcal{Z}$ seen as a $\mathbb{R}$-vector space and the property of composition of a symplectic Fourier transform by a symplectomorphism (see Appendix C). The boundedness from $\mathcal{D}\left(\langle\mathrm{N}\rangle^{\mathrm{m} / 2}\right)$ to $\mathcal{H}$ is deduced from the facts 
that the Fourier transform of $\mathrm{b}$ involves only derivatives of the delta function of order smaller or equal to $m$ and that a derivation of the Weyl operator gives at worse a field factor which is controlled by $\langle\mathrm{N}\rangle^{1 / 2}$.

\subsection{Action of Bogoliubov transformations on Wick quantization of poly- nomials in finite dimension}

Proposition 8.8. Let $\mathrm{b} \in \mathcal{P}_{\leq \mathrm{m}}(\mathcal{Z})$ with $\mathrm{m} \geq 2$ be a polynomial on a finite-dimensional Hilbert space $\mathcal{Z}$. Let $\mathrm{T}$ be an implementable symplectomorphism with implementation Upreserving the domain $\mathcal{D}\left(\langle\mathrm{N}\rangle^{\mathrm{m} / 2}\right)$. Then

$$
\mathrm{U}^{*} \mathrm{~b}^{\text {Wick}} \mathrm{U}=\left(\mathrm{e}^{\frac{\varepsilon}{2} \wedge[\mathrm{T}]}\left[\mathrm{b}\left(\mathrm{T}^{*} \cdot\right)\right]\right)^{\text {Wick }}
$$

as a continuous operator from $\mathcal{D}\left(\langle\mathrm{N}\rangle^{\mathrm{m} / 2}\right)$ to $\mathcal{H}$, where $\Lambda[\mathrm{T}]$ is defined as in Theorem 8.5 ,

Proof. We search the polynomial $c$ such that $\mathrm{U}^{*} \mathrm{~b}^{\text {Wick }} \mathrm{U}=\mathrm{c}^{\text {Wick }}$. In finite dimension for polynomials we can use the well known deconvolution formula

$$
c^{\text {Wick }}=\left(c * \frac{1}{(\pi \varepsilon / 2)^{\mathrm{d}}} e^{\frac{|z|^{2}}{\varepsilon / 2}}\right)^{\text {Weyl }} .
$$

By Proposition 8.7 we boil down to search for a polynomial $\mathrm{c}$ such that

$$
\left(\mathrm{b} * \frac{1}{(\pi \varepsilon / 2)^{\mathrm{d}}} e^{\frac{|z|^{2}}{\varepsilon / 2}}\right)\left(\mathrm{T}^{*} \cdot\right)=\mathrm{c} * \frac{1}{(\pi \varepsilon / 2)^{\mathrm{d}}} e^{\frac{|z|^{2}}{\varepsilon / 2}} .
$$

Using symplectic Fourier transform (see appendix $[$ ) and its properties with respect to convolution, composition with symplectomorphisms and Gaussians, we get

$$
\begin{aligned}
\mathcal{F}^{\sigma} \mathrm{c} & =\left[\mathcal{F}^{\sigma} \mathrm{b}\left(\mathrm{T}^{*} \cdot\right)\right] \times\left[\mathcal{F}^{\sigma}\left(\frac{e^{\frac{|z|^{2}}{\varepsilon / 2}}}{(\pi \varepsilon / 2)^{\mathrm{d}}}\right)\left(\mathrm{T}^{*} \cdot\right)\right] \times\left[\mathcal{F}^{\sigma}\left(\frac{e^{-\frac{|z|^{2}}{\varepsilon / 2}}}{(\pi \varepsilon / 2)^{\mathrm{d}}}\right)\right] \\
& =e^{\frac{\pi^{2} \varepsilon\left(\left|\mathrm{T}^{*} \cdot\right|^{2}-|\cdot|^{2}\right)}{2}} \times \mathcal{F}^{\sigma} \mathrm{b}\left(\mathrm{T}^{*} \cdot\right) .
\end{aligned}
$$

Writing $T=L+A$ with $L$ the $\mathbb{C}$-linear and $A$ the $\mathbb{C}$-antilinear part of $T$ we obtain

$$
\begin{aligned}
\left|\mathrm{T}^{*} z\right|^{2}-|z|^{2} & =\left\langle\mathrm{L}^{*} z, \mathrm{~L}^{*} z\right\rangle+\left\langle\mathrm{A}^{*} z, \mathrm{~A}^{*} z\right\rangle+\left\langle\mathrm{L}^{*} z, \mathrm{~A}^{*} z\right\rangle+\left\langle\mathrm{A}^{*} z, \mathrm{~L}^{*} z\right\rangle-\langle z, z\rangle \\
& =\left\langle z, \mathrm{LL}^{*} z\right\rangle+\left\langle z, A A^{*} z\right\rangle+\left\langle\mathrm{LA}^{*} z, z\right\rangle+\left\langle z, \mathrm{LA}^{*} z\right\rangle-\langle z, z\rangle \\
& =\left\langle z, 2 A A^{*} z\right\rangle+\left\langle v, z^{\vee^{2}}\right\rangle+\left\langle z^{2}, v\right\rangle
\end{aligned}
$$

with $v \in \otimes^{2} \mathcal{Z}$ the vector such that for all $z_{1}, z_{2} \in \mathcal{Z},\left\langle z_{1} \otimes z_{2}, v\right\rangle=\left\langle z_{1}, \mathrm{LA}^{*} z_{2}\right\rangle$. By Fourier transforming again, we get

$$
\pi^{2} \mathcal{F}^{\sigma}\left[\left(\left|T^{*} \cdot\right|^{2}-|\cdot|^{2}\right) \times \cdot\right] \mathcal{F}^{\sigma} \mathrm{c}=\operatorname{Tr}\left[-2 A A^{*} \partial_{\bar{z}} \partial_{z} c(z)\right]+\left\langle v\left|\partial_{\bar{z}}^{2} \mathrm{c}(z)+\partial_{z}^{2} \mathrm{c}(z)\right| v\right\rangle
$$

as the $\mathbb{C}$-linear and $\mathbb{C}$-antilinear parts behave differently under Fourier transform (the $\mathbb{C}$-linear part has a minus sign added, see appendix (C). We then obtain the claimed result. 


\subsection{Extension to infinite dimension on a "cylindrical" class of polyno- mials}

Theorem 8.9. Let $\uparrow$ be symplectomorphism of the form $\uparrow=e^{\mathrm{c} \rho}$, with $\mathrm{c}$ a conjugation and $\rho$ a positive, self-adjoint, Hilbert-Schmidt operator commuting with c. Let $\left(\xi_{\mathbf{j}}\right)_{\mathbf{j} \in \mathbb{N}}$ a Hilbert basis in which $\rho$ is diagonal. Let $\pi_{\mathrm{K}}$ be the orthogonal projection on the finite-dimensional space $\mathcal{Z}_{\mathrm{K}}=$ $\operatorname{Vect}\left(\left\{\xi_{j}\right\}_{j \leq K}\right)$.

Then for any polynomial $\mathrm{b}$ in $\mathcal{P}_{\mathrm{m}}(\mathcal{Z})$ with $\mathrm{m} \geq 2$ and any integer $\mathrm{K}$

$$
\widehat{\mathrm{U}}^{*} \mathrm{~b}_{\mathrm{K}}^{\text {Wick}} \hat{\mathrm{U}}=\left(e^{\frac{\varepsilon}{2} \Lambda[\hat{\mathrm{\top}}]}\left[\mathrm{b}_{\mathrm{K}}\left(\hat{\mathrm{\top}}^{*} \cdot\right)\right]\right)^{\text {Wick }}
$$

as continuous operators from $\mathcal{D}\left(\langle\mathrm{N}\rangle^{\frac{\mathrm{m}}{2}}\right)$ to $\mathcal{H}$ where $\mathrm{b}_{\mathrm{K}}(z)=\mathrm{b}\left(\pi_{\mathrm{K}} z\right)$.

Proof. We first remark that, with $\mathrm{Q}(z)=\mathfrak{I}\langle c \rho z, z\rangle, e^{-i \mathrm{Q}^{W_{i c k}} / \varepsilon}$ is a Bogoliubov implementer of $\uparrow$ as it can be seen using Proposition 8.2 and the Hilbert-Schmidt property of $\rho$. We define $\rho_{\mathrm{L}}=\rho \pi_{\mathrm{L}}$, $\mathrm{T}_{\mathrm{L}}=\mathrm{\top}_{\pi_{\mathrm{L}}}$ and the operator $\mathrm{Q}_{\mathrm{L}}(z)^{\text {Wick }}=\mathfrak{I}\left\langle c \rho_{\mathrm{L}} z, z\right\rangle^{\text {Wick }}$. We use the identification $\mathcal{H}=\Gamma_{\mathrm{s}}\left(\mathcal{Z}_{\mathrm{L}}\right) \otimes$ $\Gamma_{\mathrm{s}}\left(\mathcal{Z}_{\mathrm{L}}^{\perp}\right)$ and observe that on $\Gamma_{\mathrm{s}}\left(\mathcal{Z}_{\mathrm{L}}\right) \otimes\left\{\Omega^{\mathcal{Z}_{\mathrm{L}}^{\perp}}\right\}, e^{-i \mathrm{Q}^{\text {Wick }} / \varepsilon}=e^{-i \mathrm{Q}_{\mathrm{L}}^{\text {Wick }} / \varepsilon}$. For $\mathrm{K} \leq \mathrm{L}$ we obtain on $\Gamma_{\mathrm{S}}\left(\mathcal{Z}_{\mathrm{L}}\right) \otimes\left\{\Omega^{\mathcal{Z}_{\mathrm{L}}^{\perp}}\right\}$

$$
\widehat{\mathrm{u}}_{\mathrm{L}}^{*} \mathrm{~b}_{\mathrm{K}}^{\text {Wick}} \widehat{\mathrm{U}}_{\mathrm{L}}=\left(e^{\frac{\varepsilon}{2} \wedge\left[\hat{\mathrm{T}}_{\mathrm{L}}\right]}\left[\mathrm{b}_{\mathrm{K}}\left(\hat{\mathrm{T}}_{\mathrm{L}}^{*} \cdot\right)\right]\right)^{\text {Wick }}
$$

by Proposition 8.8, with $\widehat{\mathrm{U}}_{\mathrm{L}}=e^{-i \mathrm{Q}_{\mathrm{L}}^{\text {Wick }} / \varepsilon}$. But on this domain it is the same as

$$
\widehat{\mathrm{u}}^{*} \mathrm{~b}_{\mathrm{K}}^{\text {Wick}} \mathfrak{u}=\left(e^{\frac{\varepsilon}{2} \wedge[\hat{\top}]}\left[\mathrm{b}_{\mathrm{K}}\left(\hat{\mathrm{T}}^{*} \cdot\right)\right]\right)^{\text {Wick }}
$$

with $\hat{\mathrm{U}}=e^{-i \mathrm{Q}^{\text {Wick }} / \varepsilon}$. We thus get an equality on $\cup_{\mathrm{L}} \Gamma_{\mathrm{S}}\left(\mathcal{Z}_{\mathrm{L}}\right)$, and by continuity of the involved operators from $\mathcal{D}\left(\langle\mathrm{N}\rangle^{\frac{\mathrm{m}}{2}}\right)$ to $\mathcal{H}$ we get the expected result.

We will first show that Formula (8.1) apply in particular to a well chosen class of cylindrical polynomials, and then extend it by density to every polynomial.

\subsection{Extension to general polynomials}

We split the proof of Formula (8.1) for general polynomials into several lemmata and propositions.

Lemma 8.1. Let $\left(\xi_{\mathrm{j}}\right)_{\mathbf{j} \in \mathbb{N}}$ be a Hilbert basis of $\mathcal{Z}, \pi_{\mathrm{m}}$ be the orthogonal projector on $\mathcal{Z}_{\mathrm{m}}=$ $\operatorname{Vect}\left(\left\{\xi_{\mathrm{j}}\right\}_{\mathrm{j} \leq \mathrm{m}}\right)$. Let $\mathrm{b}$ be a polynomial in $\mathcal{P}_{\mathrm{p}, \mathrm{q}}(\mathcal{Z})$ and define $\mathrm{b}_{\mathrm{K}}=\mathrm{b}\left(\pi_{\mathrm{K}} \cdot\right)$. Then $\left(\widetilde{\mathrm{b}_{\mathrm{K}}}\right)_{\mathrm{K} \in \mathbb{N}}$ is bounded and

$$
\tilde{\mathrm{b}}=w-\lim _{j \rightarrow \infty} \widetilde{b_{K}}
$$

To formulate more clearly some convergence results we need some extra definitions. 
Definition 8.10. We define the spaces

$$
\mathcal{L}_{\mathrm{p}, \mathrm{q}}^{\vee}(\mathcal{Z})=\mathcal{L}\left(\mathcal{Z}^{\vee p}, \mathcal{Z}^{\vee \mathfrak{q}}\right), \quad \mathcal{L}_{\mathrm{m}}^{\vee}=\bigoplus_{\mathrm{p}+\mathrm{q}=\mathrm{m}} \mathcal{L}_{\mathrm{p}, \mathrm{q}}^{\vee} \quad \text { and } \quad \mathcal{L}_{\leq \mathrm{m}}^{\vee}=\bigoplus_{\mathrm{m}^{\prime} \leq \mathrm{m}} \mathcal{L}_{\mathrm{m}^{\prime}}^{\vee}
$$

corresponding to $\mathcal{P}_{\mathrm{p}, \mathrm{q}}(\mathcal{Z}), \mathcal{P}_{\mathrm{m}}(\mathcal{Z})$ and $\mathcal{P}_{\leq \mathrm{m}}(\mathcal{Z})$.

Let $\mathrm{b}=\sum_{\mathrm{p}, \mathrm{q}} \mathrm{b}_{\mathrm{p}, \mathrm{q}}$ be a polynomial, with $\mathrm{b}_{\mathrm{p}, \mathrm{q}} \in \mathcal{P}(\mathcal{Z})$. We note $\tilde{\mathrm{b}}=\left(\widetilde{\mathrm{b}_{\mathrm{p}, \mathrm{q}}}\right) \in \bigoplus_{\mathrm{p}, \mathrm{q}} \mathcal{L}_{\mathrm{p}, \mathrm{q}}^{\vee}(\mathcal{Z})$.

The norm of $\tilde{\mathrm{b}}=\left(\widetilde{\mathrm{b}_{\mathrm{p}, \mathrm{q}}}\right) \in \mathcal{L}_{\leq \mathrm{m}}^{\vee}(\mathcal{Z})$ is $\|\tilde{\mathrm{b}}\|_{\mathcal{L}_{\leq m}^{\vee}(\mathcal{Z})}=\sum_{\mathrm{p}, \mathrm{q}}\left\|\widetilde{\mathrm{b}_{\mathrm{p}, \mathrm{q}}}\right\|_{\mathcal{L}\left(\bigvee^{\mathrm{p}} \mathcal{Z}, \bigvee^{q} \mathcal{Z}\right)}$.

A sequence $\left(\tilde{b}_{K}\right)_{K \in \mathbb{N}}$ of elements of $\mathcal{L}_{\leq \mathrm{m}}(\mathcal{Z})$ converges weakly to $\tilde{\mathrm{b}}$ in $\mathcal{L}_{\leq \mathrm{m}}(\mathcal{Z})$ if $\tilde{\mathrm{b}}_{\mathrm{K}_{\mathrm{p}, \mathrm{q}}}$ converges weakly to $\tilde{b}_{p, q}$ for every $p$ and $q$ as $K \rightarrow+\infty$.

Lemma 8.2. Let $\mathrm{T}$ be an operator in $\mathcal{X}(\mathcal{Z}),\left(\mathrm{b}_{\mathrm{K}}\right)_{\mathrm{K} \in \mathbb{N}}$ and $\mathrm{b}$ be polynomials in $\mathcal{P}_{\mathrm{m}}(\mathcal{Z})$ such that $\left(\tilde{\mathrm{b}}_{\mathrm{K}}\right)_{\mathrm{K} \in \mathbb{N}}$ converges weakly to $\tilde{\mathrm{b}}$. Then $\mathrm{b}_{\mathrm{K}}(\mathrm{T} \cdot)$ and $\mathrm{b}(\mathrm{T} \cdot)$ are in $\mathcal{P}_{\mathrm{m}}(\mathcal{Z})$ and $\widetilde{\mathrm{b}_{\mathrm{K}}(\mathrm{T} \cdot)}$ converges weakly to $\mathrm{b}(\mathrm{T} \cdot)$.

Lemma 8.3. Let $\mathrm{T}$ be an operator in $\mathcal{X}(\mathcal{Z}),\left(\mathrm{b}_{\mathrm{K}}\right)_{\mathrm{K} \in \mathbb{N}}$ and $\mathrm{b}$ be polynomials in $\mathcal{P}_{\mathrm{m}}(\mathcal{Z})$ such that $\left(\tilde{\mathrm{b}}_{\mathrm{K}}\right)_{\mathrm{K} \in \mathbb{N}}$ is bounded and converges weakly to $\tilde{\mathrm{b}}$. Then $\left(\mathrm{e}^{\overline{\frac{\varepsilon}{2} \wedge[\mathrm{T}]}} \mathrm{b}_{\mathrm{K}}\right)_{\mathrm{K} \in \mathbb{N}}$ converges weakly to $\overline{\mathrm{e}^{\frac{\varepsilon}{2} \wedge[\mathrm{T}]} \mathrm{b}}$.

Proof. It is enough to show that weak convergence is preserved by the action of $\Lambda[T]$. But, for any polynomial $b$,

$$
\widetilde{\Lambda[T] b}=\operatorname{Tr}_{1}\left[\left(-2 A^{*} A \otimes I_{\mathcal{Z} \vee q-1}\right) \tilde{b}\right]+\left(\langle v| \vee I_{\mathcal{Z} \vee q-2}\right) \tilde{b}+\tilde{b}\left(|v\rangle \vee I_{\mathcal{Z} \vee p-2}\right),
$$

where $\operatorname{Tr}_{1}$ is the partial trace on the first $\mathcal{Z}$ subspace on the left and any direction on the right (so that if $\tilde{b} \in \mathcal{L}_{\mathfrak{p}, q}^{\vee}(\mathcal{Z})$, then $\operatorname{Tr}_{1}\left[\left(-2 A^{*} A \otimes I_{\mathcal{Z}^{\vee q-1}}\right) \tilde{b}\right]$ is in $\left.\mathcal{L}_{\mathbf{p}-1, q-1}^{\vee}(\mathcal{Z})\right)$. With this formula the preservation of the weak convergence is clear.

Proposition 8.11. Let $\mathrm{b}$ and $\left(\mathrm{b}_{\mathrm{K}}\right)_{\mathrm{K} \in \mathbb{N}}$ be Wick polynomials in $\mathcal{P}_{\mathrm{p}, \mathrm{q}}(\mathcal{Z})$ such that $w-\lim \tilde{b}_{\mathrm{K}}=\tilde{\mathrm{b}}$. Then

$$
w-\lim _{\mathrm{K}}\left(\mathrm{b}_{\mathrm{K}}-\mathrm{b}\right)^{\text {Wick }}\langle\mathrm{N}\rangle^{-\frac{\mathrm{p}+\mathrm{q}}{2}}=0 .
$$

Proposition 8.12. Let $\mathrm{b}$ and $\left(\mathrm{b}_{\mathrm{K}}\right)_{\mathrm{K} \in \mathbb{N}}$ be Wick polynomials in $\mathcal{P}_{\mathrm{p}, \mathrm{q}}(\mathcal{Z})$ such that $w-\lim \tilde{\mathrm{b}}_{\mathrm{K}}=\tilde{\mathrm{b}}$. Let $\mathrm{U}$ be a unitary operator on the Fock space $\mathcal{H}$ such that, for all $k \geq 2,\langle\mathrm{~N}\rangle^{\frac{k}{2}} \mathrm{U}\langle\mathrm{N}\rangle^{-\frac{k}{2}}$ is a bounded operator. Then

$$
w-\lim _{\mathrm{K}} \mathrm{U}^{*}\left(\mathrm{~b}_{\mathrm{K}}-\mathrm{b}\right)^{\text {Wick }} \mathrm{U}\langle\mathrm{N}\rangle^{-\frac{\mathrm{m}^{\prime}}{2}}=0
$$

with $\mathrm{m}^{\prime}=\max (\mathrm{m}, 2), \mathrm{m}=\mathrm{p}+\mathrm{q}$.

Proposition 8.13. Let $\mathrm{T}$ be an implementable symplectomorphism with Bogoliubov implementer $\mathrm{U}$. Then for any polynomial $\mathrm{b}$ in $\mathcal{P}_{\leq \mathrm{m}}(\mathcal{Z}), \mathrm{m} \geq 2$,

$$
\mathrm{U}^{*} \mathrm{~b}^{\text {Wick }} \mathrm{U}=\left(\mathrm{e}^{\frac{\varepsilon}{2} \wedge[\mathrm{T}]}\left[\mathrm{b}\left(\mathrm{T}^{*} \cdot\right)\right]\right)^{\text {Wick }}
$$

as continuous operators from $\mathcal{D}\left(\langle\mathrm{N}\rangle^{\frac{\mathrm{m}}{2}}\right)$ to $\mathcal{H}$. 
Proof. From the results 8.1 to 8.12 we deduce that Proposition 8.13 holds for symplectomorphisms of the form $\hat{\top}=e^{c \rho}$, with $c$ a conjugation and $\rho$ a positive, self-adjoint, Hilbert-Schmidt operator commuting with $\mathrm{c}$.

By Theorem A.8 this assumption on the form of $\uparrow$ is not restrictive. Indeed, if $\mathrm{T}=\mathrm{u} \uparrow$ with $u$ unitary and $\widehat{U}$ is a Bogoliubov implementer for $\uparrow$, then $\mathrm{U}=\widehat{\mathrm{U}} \Gamma\left(\mathrm{u}^{*}\right)$ is a Bogoliubov implementer for $\mathrm{T}$ and

$$
\Gamma(\mathrm{u})\left(\mathrm{e}^{\frac{\varepsilon}{2} \wedge[\hat{\mathrm{T}}]}\left[\mathrm{b}\left(\hat{\mathrm{T}}^{*} \cdot\right)\right]\right)^{\text {Wick }} \Gamma\left(\mathrm{u}^{*}\right)=\left(\mathrm{e}^{\frac{\varepsilon}{2} \wedge[\mathrm{T}]}\left[\mathrm{b}\left(\mathrm{T}^{*} \cdot\right)\right]\right)^{\text {Wick }} .
$$

Indeed for any polynomial $\mathrm{c}$, and operator $\varphi$ in $\mathcal{X}(\mathcal{Z}), \Gamma(\varphi) \mathrm{c}^{\text {Wick }} \Gamma\left(\varphi^{*}\right)=\mathrm{c}\left(\varphi^{*} \cdot\right)^{\text {Wick }}$ and

$$
\Lambda[\hat{\top}]^{k}\left[b\left(\hat{\top}^{*} \cdot\right)\right]\left(u^{*} \cdot\right)=\Lambda[u \uparrow]^{k}\left[b\left(\uparrow^{*} u^{*} \cdot\right)\right]
$$

as can be checked using that $L=u \hat{L}$ and $A=u \hat{A}$ ( $L, \hat{L}$ and $A, \hat{A}$ denote respectively the $\mathbb{C}$-linear and $\mathbb{C}$-antilinear parts of $\mathrm{T}$ and $\hat{\mathrm{T}})$. This achieves the proof.

\subsection{An evolution formula for the Wick symbol}

We can now prove Theorem 3.2 .

Proof. We only need to apply propositions 8.2 and 8.13 with $T=-i \varphi(0, t) i=L^{*}(t, 0)+A^{*}(t, 0)$ (with $\varphi(t, 0)=L(t, 0)+A(t, 0)$ ). We remark that for any symplectomorphism $\mathrm{T},(-\mathrm{iTi})^{*}=\mathrm{T}^{-1}$ so that $(-i \varphi(0, t) i)^{*}=\varphi(t, 0)$ and thus we get the result.

\subsection{Estimates}

We now give estimates for the different terms of the expansion of the symbol.

Proposition 8.14. Let $\mathrm{T}=\mathrm{L}+\mathrm{A}$ be an implementable symplectomorphism with $\mathrm{L} \mathbb{C}$-linear and $\mathrm{A}$ $\mathbb{C}$-antilinear. Then the operator $\Lambda[T]$ defined on $\mathcal{P}(\mathcal{Z})$ by

$$
\Lambda[T] c(z)=\operatorname{Tr}\left[-2 A A^{*} \partial_{\bar{z}} \partial_{z} c\right]+\left\langle v\left|\partial_{\bar{z}}^{2} c(z)+\partial_{z}^{2} c(z)\right| v\right\rangle,
$$

with $v \in \otimes^{2} \mathcal{Z}$ the vector such that for all $z_{1}, z_{2} \in \mathcal{Z},\left\langle z_{1} \otimes z_{2}, v\right\rangle=\left\langle z_{1}\right.$, LA* $\left.z_{2}\right\rangle$ is such that, for $\mathrm{c}$ in $\mathcal{P}_{\mathrm{m}}(\mathcal{Z})$

$$
\|\wedge[\mathrm{T}] \mathrm{c}\|_{\mathcal{P}_{\mathrm{m}-2}(\mathcal{Z})} \leq 2\|\mathrm{~T}\|_{\mathcal{X}(\mathcal{Z})}\|\mathrm{A}\|_{\mathcal{L}_{2}^{\mathrm{a}}(\mathcal{Z})}\|\mathrm{c}\|_{\mathcal{P}_{\mathrm{m}}(\mathcal{Z})}
$$

Proof. We only have to remark that for any polynomial $\mathrm{c}$ in $\mathcal{P}_{\mathrm{p}, \mathrm{q}}(\mathcal{Z})$ the following estimates hold

$$
\left\|\operatorname{Tr}\left[\mathrm{B} \partial_{\bar{z}} \partial_{\mathcal{z}} \mathrm{c}(z)\right]\right\|_{\mathrm{q}-1 \leftarrow \mathrm{p}-1} \leq\|\mathrm{B}\|_{\mathcal{L}_{1}(\mathcal{Z})}\|\mathrm{c}\|_{\mathrm{q} \leftarrow \mathrm{p}}
$$

for any trace class operator $\mathrm{B}$, and

$$
\|\langle v| \partial_{\bar{z}}^{2} \mathrm{c}(z)\left\|_{\mathrm{q}-2 \leftarrow \mathrm{p}} \leq\right\| v\left\|_{\mathrm{V}^{2} \mathcal{Z}}\right\| \mathrm{c} \|_{\mathrm{q} \leftarrow \mathrm{p}}
$$

and that $\|v\|_{\bigvee^{2} \mathcal{Z}}=\left\|L A^{*}\right\|_{\mathcal{L}_{2}^{\mathrm{a}}(\mathcal{Z})} \leq\|\mathrm{L}\|_{\mathcal{L}(\mathcal{Z})}\|A\|_{\mathcal{L}_{2}^{\mathrm{a}}(\mathcal{Z})}$. The same estimate holds for $\partial_{z}^{2} \mathrm{c}(z)|v\rangle$. 
We apply this result to the expression given in the theorem 3.2

Proposition 8.15. Let $\left(\mathrm{Q}_{\mathrm{t}}\right)_{\mathrm{t}}$ be a continuous one parameter family of quadratic polynomials, $\varphi$ the classical flow associated to $\left(\mathrm{Q}_{\mathrm{t}}\right)_{\mathrm{t}}$, and $\Lambda^{\mathrm{t}}$ the operator defined in theorem 3.2 Then, for $\mathrm{b}$ in $\mathcal{P}_{\leq m}(\mathcal{Z})$

$$
\left\|e^{\frac{\varepsilon}{2} \Lambda^{t}}(\mathrm{~b} \circ \varphi(t, 0))\right\|_{\mathcal{P}(\mathcal{Z})} \leq\|\mathrm{b}\|_{\mathcal{P}(\mathcal{Z})}\|\varphi(t, 0)\|_{\mathcal{X}(\mathcal{Z})}^{m} \sum_{k=0}^{m} \frac{1}{k !}\left(\varepsilon\|\varphi(t, 0)\|_{\mathcal{X}(\mathcal{Z})}\|\mathrm{A}(\mathrm{t}, 0)\|_{\mathcal{L}_{2}^{a}(\mathcal{Z})}\right)^{k}
$$

where $\mathrm{A}$ is the $\mathbb{C}$-antilinear part of $\varphi$.

Proof. It is enough to combine the propositions 4.4 and 8.14

Remark 8. The norm $\|\varphi(t, 0)\|_{\mathcal{X}(\mathcal{Z})}$ is bigger than 1 as for any symplectic transformation $T=$ $\mathrm{L}+\mathrm{A}$ with $\mathrm{L} \mathbb{C}$-linear and $\mathrm{A} \mathbb{C}$-antilinear, $\mathrm{L}^{*} \mathrm{~L}=\mathrm{I}_{\mathcal{Z}}+\mathrm{A}^{*} \mathrm{~A} \geq \mathrm{I}_{\mathcal{Z}}$ (see proposition A.4) and thus $\|\mathrm{T}\|_{\mathcal{X}(\mathcal{Z})} \geq\|\mathrm{L}\|_{\mathcal{L}(\mathcal{Z})} \geq 1$.

\section{Appendices}

\section{A $\mathbb{R}$-linear symplectic transformations}

In this part we adapt and recall some results of [26] to fit our needs.

Let $(\mathcal{Z},\langle\cdot, \cdot\rangle)$ be a separable Hilbert space over the complex numbers field $\mathbb{C}$. The scalar products is linear with respect to the right variable and antilinear with respect to the left variable. We note $\operatorname{Aut}_{\mathbb{R}}(\mathcal{Z})$ the group of $\mathbb{R}$-linear continuous automorphisms on $\mathcal{Z}$. We define a symplectic form $\sigma$ on $\mathcal{Z}$ by

$$
\sigma\left(z_{1}, z_{2}\right):=\mathfrak{I}\left\langle z_{1}, z_{2}\right\rangle .
$$

Definition A.1. A $\mathbb{R}$-linear automorphism $\mathrm{T}$ is a symplectomorphism if it preserves the symplectic form, i.e. if

$$
\forall z_{1}, z_{2} \in \mathcal{Z}, \quad \sigma\left(T z_{1}, T z_{2}\right)=\sigma\left(z_{1}, z_{2}\right) .
$$

We note $\operatorname{Sp}_{\mathbb{R}}(\mathcal{Z})$ the set of symplectic transformations over the Hilbert space $\mathcal{Z}$. It is a subgroup of $\operatorname{Aut}_{\mathbb{R}}(\mathcal{Z})$.

Proposition A.2. A $\mathbb{R}$-linear application $\mathrm{T}: \mathcal{Z} \rightarrow \mathcal{Z}$ can be written as a sum of two applications respectively $\mathbb{C}$-linear and $\mathbb{C}$-antilinear in a unique way:

$$
\mathrm{T}=\frac{\mathrm{T}-\mathrm{iTi}}{2}+\frac{\mathrm{T}+\mathrm{iTi}}{2} .
$$

Definition A.3. Let $A$ be a (bounded) $\mathbb{C}$-antilinear operator on the Hilbert space $\mathcal{Z}$. We define its adjoint $A^{*}$ as the only antilinear operator such that

$$
\forall z_{1}, z_{2} \in \mathcal{Z}, \quad\left\langle z_{1}, A z_{2}\right\rangle=\left\langle z_{2}, A^{*} z_{1}\right\rangle .
$$


Let $\mathrm{T}=\mathrm{L}+\mathrm{A}=\mathcal{Z} \rightarrow \mathcal{Z}$ be a $\mathbb{R}$-linear application with $\mathrm{L} \mathbb{C}$-linear and $\mathrm{A} \mathbb{C}$-antilinear. The adjoint $\mathrm{T}^{*}$ of $\mathrm{T}$ is defined by $\mathrm{T}^{*}=\mathrm{L}^{*}+\mathrm{A}^{*}$.

Proposition A.4. Let $\mathrm{T}=\mathrm{L}+\mathrm{A}$ be a $\mathbb{R}$-linear automorphism with $\mathrm{L} \mathbb{C}$-linear and $\mathrm{A} \mathbb{C}$-antilinear, then the following conditions are equivalent.

(1) $\mathrm{L}+\mathrm{A}$ is a symplectomorphism.

(2) $\left(L^{*}-A^{*}\right)(L+A)=I_{\mathcal{Z}}$.

(3) $\left(L^{*}+A^{*}\right)(L-A)=I_{\mathcal{Z}}$.

(4) $L^{*} L-A^{*} A=I_{\mathcal{Z}}$ and $L^{*} A=A^{*} L$.

(5) $\mathrm{L}^{*}-\mathrm{A}^{*}$ is a symplectomorphism.

(6) $\mathrm{L}-\mathrm{A}$ is a symplectomorphism.

(7) $\mathrm{LL}^{*}-\mathrm{A} A^{*}=\mathrm{I}_{\mathcal{Z}}$ and $\mathrm{A}^{*} \mathrm{~L}=\mathrm{L}^{*} \mathrm{~A}$.

Proof. (1) $\Leftrightarrow$ (2) Let $\mathrm{T}=\mathrm{L}+\mathrm{A}$ a symplectomorphism, for all $z_{1}, z_{2} \in \mathcal{Z}$,

$$
\begin{aligned}
\sigma\left(z_{1}, z_{2}\right)=\mathfrak{I}\left\langle z_{1}, z_{2}\right\rangle & =\mathfrak{I}\left\langle(\mathrm{L}+\mathrm{A}) z_{1}, \mathrm{~T} z_{2}\right\rangle \\
& =\mathfrak{I}\left(\left\langle z_{1}, \mathrm{~L}^{*} \mathrm{~T} z_{2}\right\rangle+\overline{\left\langle z_{1}, A^{*} \mathrm{~T} z_{2}\right\rangle}\right) \\
& =\mathfrak{I}\left\langle z_{1},\left(\mathrm{~L}^{*}-A^{*}\right) \mathrm{T} z_{2}\right\rangle .
\end{aligned}
$$

Replacing $z_{1}$ by $i z_{1}$ we get the same relation with a real part instead of an imaginary part and finally

$$
\left\langle z_{1},\left[\left(L^{*}-A^{*}\right)(L+A)-I_{\mathcal{Z}}\right] z_{2}\right\rangle=0
$$

and this in turn implies $\left(L^{*}-A^{*}\right)(L+A)=I_{\mathcal{Z}}$. We can reverse the order of these calculations in order to obtain the first equivalence.

(2) $\Leftrightarrow(3)$ The $\mathbb{C}$-linearity and antilinearity properties of $L$ and $A$ give

$$
\left(L^{*}-A^{*}\right)(L+A) i=i\left(L^{*}+A^{*}\right)(L-A)
$$

so that we get the equivalent condition (3).

((2) and (3)) $\Leftrightarrow(4)$ The sum and the difference of the equations of (2) and (3) give (4) and the sum and difference of the equations in (4) give (2) and (3).

(1) $\Leftrightarrow(5)$ From (1) and (3) we know that the inverse of a symplectomorphism $T=L+A$ is $\mathrm{T}^{-1}=\mathrm{L}^{*}-A^{*}$ which is necessarily a symplectomorphism too, and thus (1) $\Rightarrow(5)$. We get (5) $\Rightarrow(1)$ exchanging $\mathrm{T}$ and $\mathrm{T}^{-1}$.

$(1) \Leftrightarrow(6) \Leftrightarrow(7)$ is easily deduced from the previous equivalences. 
Proposition A.5. Let $\mathrm{T}=\mathrm{L}+\mathrm{A}$ be a symplectomorphism with $\mathrm{L} \mathbb{C}$-linear and $\mathrm{A} \mathbb{C}$-antilinear, then $\mathrm{L}$ is invertible.

Proof. From Proposition A.4 we get

$$
L^{*} L=I_{\mathcal{Z}}+A^{*} A \geq I_{\mathcal{Z}} \quad \text { and } \quad L L^{*}=I_{\mathcal{Z}}+A A^{*} \geq I_{\mathcal{Z}}
$$

and thus $L$ and $L^{*}$ are one to one. As $L^{*}$ is one to one we get $\overline{\operatorname{RanL}}=\left(\operatorname{KerL}^{*}\right)^{\perp}=\{0\}^{\perp}=\mathcal{Z}$.

It is now enough to show that the range of $L$ is closed. Pick a vector $y \in \mathcal{Z}$, there is a sequence $\left(x_{n}\right) \in \mathcal{Z}^{\mathbb{N}}$ such that $\mathrm{L} x_{n} \rightarrow y$. The relation $L^{*} \mathrm{~L} \geq \mathrm{I}_{\mathcal{Z}}$ gives $\left|\mathrm{L} x_{m}-\mathrm{L} x_{n}\right| \geq\left|x_{n}-x_{m}\right|$. The left hand part of the inequality goes to 0 for $m, n \rightarrow \infty$, so that $\left(x_{n}\right)$ is a Cauchy sequence and thus converges to a limit $x$. By continuity of $L, L x=y$ and $L$ is indeed one to one.

Definition A.6. An application c from $\mathcal{Z}$ to $\mathcal{Z}$ is a conjugation if and only if it satisfies the following conditions.

(1) $\mathrm{c}$ is $\mathbb{R}$-linear.

(2) $c^{2}=I_{\mathcal{Z}}$

(3) For all $z_{1}, z_{2}$ in $\mathcal{Z},\left\langle\mathrm{c} z_{1}, z_{2}\right\rangle=\left\langle\mathrm{c} z_{2}, z_{1}\right\rangle$.

Remark 9. It follows from the third condition in this definition that a conjugation is antilinear.

One may define different conjugations on the same Hilbert space over $\mathbb{C}$ (even for a one dimensional Hilbert space). As an example one can consider a Hilbert basis $\left(\boldsymbol{e}_{\mathfrak{j}}\right)$ and define the application $c: \sum_{j} \alpha_{j} e_{j} \mapsto \sum_{j} \overline{\alpha_{j}} e_{j}$.

Definition A.7. Let $c$ be a conjugation on the Hilbert space $\mathcal{Z}$. The real and imaginary parts of a vector $z \in \mathcal{Z}$ (with respect to the conjugation $\mathrm{c}$ ) are defined as

$$
\mathfrak{R} z:=\frac{z+c z}{2} \text { and } \Im z:=\frac{z-c z}{2 i} .
$$

They verify $z=\mathfrak{R} z+\mathfrak{i} \Im z$. The space $\mathrm{E}_{\mathbb{R}}^{c}:=\mathfrak{R} \mathcal{Z}=\mathfrak{I Z}$ is a subspace of $\mathcal{Z}$ as $\mathbb{R}$-vector space, $\langle\cdot, \cdot \cdot\rangle$ restricted to $E_{\mathbb{R}}^{c}$ is a real scalar product and $E=E_{\mathbb{R}}^{c} \oplus i E_{\mathbb{R}}^{c}$.

Let $f$ be a $\mathbb{R}$-linear application on $\mathcal{Z}$, then we can define the applications from $E_{\mathbb{R}}^{c}$ to itself

$$
\begin{array}{rlrl}
\alpha: z \mapsto \mathfrak{R} f(z), & \gamma: z \mapsto \mathfrak{R f}(\mathrm{iz}), \\
\beta: z & \mapsto \mathfrak{I f}(z), & \delta: z \mapsto \mathfrak{I f}(\mathrm{i} z) .
\end{array}
$$

Then, if $a, b \in E_{\mathbb{R}}^{c}$, then $f(a+i b)=\alpha(a)+i \beta(a)+\gamma(i b)+i \delta(i b)$, and $f$ can be represented as an application on $E_{\mathbb{R}}^{c} \times E_{\mathbb{R}}^{c}$ by the matrix

$$
\left(\begin{array}{ll}
\alpha & \gamma \\
\beta & \delta
\end{array}\right) .
$$

The following relations hold with the above sign if $f$ is $\mathbb{C}$-linear and with the below sign if $f$ is $\mathbb{C}$-antilinear: $\beta=\mp \gamma$ and $\alpha= \pm \delta$ and $f^{*}$ is represented by the matrix $\left(\begin{array}{c}\alpha^{\top} \mp \beta^{\top} \\ \beta^{\top} \pm \alpha^{\top}\end{array}\right)$. 
We want to show a reduction result for the symplectomorphisms in the spirit of the polar decomposition, in the case of an implementable symplectomorphism (see Definition 8.1 and Theorem 8.4).

Theorem A.8. Let $\mathrm{T}$ be an implementable symplectomorphism. Then

$$
\mathrm{T}=u e^{\mathrm{c} \rho}
$$

where

- $\mathrm{u}$ is a unitary operator,

- c is a conjugation,

- $\rho$ is a Hilbert-Schmidt, self-adjoint, non-negative operator commuting with c.

Remark 10. The operator $u$ is the unitary operator of the polar decomposition $L=u|L|$ of the $\mathbb{C}$-linear part of $T$. The conjugation $c$ is a specific conjugation associated with $L$ and will be constructed during the proof and $\rho=\arg \cos |\mathrm{L}|$.

Proof. Let us write $\mathrm{T}=\mathrm{L}+\mathrm{A}$ with $\mathrm{L} \mathbb{C}$-linear and $\mathrm{A} \mathbb{C}$-antilinear. With $\mathrm{L}=\mathfrak{u}|\mathrm{L}|$ the polar decomposition of $L$ we get $T=u\left(|L|+u^{*} A\right)$ so that it is enough to show the two next lemmas.

Lemma A.1. Let $(\mathrm{E},\langle\cdot, \cdot\rangle)$ be a finite-dimensional Hilbert space over $\mathbb{C}$. Let $\mathrm{f}: \mathrm{E} \rightarrow \mathrm{E}$ be a $\mathbb{C}$-antilinear application such that

$$
\mathrm{ff}^{*}=\mathrm{I}_{\mathrm{E}} \quad \text { and } \quad \mathrm{f}=\mathrm{f}^{*} .
$$

Then there exists an orthonormal basis $\left(\mathfrak{u}_{\mathfrak{j}}\right)$ of $\mathrm{E}$ such that

$$
\forall j, f\left(u_{j}\right)=u_{j} .
$$

Proof. Let us consider an arbitrary conjugation $c_{0}$ on $E$ and the $\left(\begin{array}{cc}\alpha & \beta \\ \beta & -\alpha\end{array}\right)$ "matrix" of $f$ (as a $\mathbb{R}$-linear operator) on $E=E_{\mathbb{R}}^{c_{0}} \oplus i E_{\mathbb{R}}^{c_{0}}$ identified with $E_{\mathbb{R}}^{c_{0}} \times E_{\mathbb{R}}^{c_{0}}$. The matrix associated to $f^{*}$ is $\left(\begin{array}{cc}\alpha^{\top} & \beta^{\top} \\ \beta^{\top} & -\alpha^{\top}\end{array}\right)$ so that the relation $f=f^{*}$ gives $\alpha=\alpha^{\top}$ and $\beta=\beta^{\top}$. From $f f^{*}=I_{E}$ we deduce $\alpha^{2}+\beta^{2}=\operatorname{Id}$ and $\alpha \beta=\beta \alpha$. We can thus diagonalize simultaneously $\alpha$ and $\beta$, and so in a convenient basis of $E_{\mathbb{R}}^{c_{0}}$ the matrix of $f$ is of the form

$$
\left(\begin{array}{ccc|ccc}
\ddots & & 0 & \ddots & & 0 \\
& \lambda_{j}^{\alpha} & & & \lambda_{j}^{\beta} & \\
0 & & \ddots & 0 & & \ddots \\
\hline \ddots & & 0 & \ddots & & 0 \\
& \lambda_{j}^{\beta} & & & -\lambda_{j}^{\alpha} & \\
0 & & \ddots & 0 & & \ddots
\end{array}\right) .
$$


We can thus confine ourself to the case of a space $E$ of complex dimension 1 and of $f$ with a matrix of the form $\left(\begin{array}{cc}\alpha & \beta \\ \beta & -\alpha\end{array}\right)$ with $\alpha$ and $\beta$ real numbers. We search a normalized vector $z=\left(\begin{array}{c}\cos \theta \\ \sin \theta\end{array}\right)=\left(\begin{array}{l}x \\ y\end{array}\right)$ and a real $\lambda$ such that $f(z)=\lambda z$, i.e.

$$
\begin{aligned}
\lambda\left(\begin{array}{l}
x \\
y
\end{array}\right) & =\left(\begin{array}{c}
\alpha x+\beta y \\
-\alpha y+\beta x
\end{array}\right)=\left(\begin{array}{cc}
x & y \\
-y & x
\end{array}\right)\left(\begin{array}{l}
\alpha \\
\beta
\end{array}\right) \\
& =\sqrt{\alpha^{2}+\beta^{2}}\left(\begin{array}{cc}
\cos \theta & \sin \theta \\
-\sin \theta & \cos \theta
\end{array}\right)\left(\begin{array}{c}
\cos \phi \\
\sin \phi
\end{array}\right)=\sqrt{\alpha^{2}+\beta^{2}}\left(\begin{array}{l}
\cos (\phi-\theta) \\
\sin (\phi-\theta)
\end{array}\right)
\end{aligned}
$$

so that if we choose $\theta$ such that $\phi-\theta=\theta$ we get the desired result with $\lambda=\sqrt{\alpha^{2}+\beta^{2}}$. Finally, from $\mathrm{ff}^{*}=\mathrm{I}_{\mathrm{E}}$ we deduce that $\lambda=1$ and the result follows.

Lemma A.2. Let $\mathrm{T}=\mathrm{L}+\mathrm{A}$ be an implementable symplectomorphism with $\mathrm{L} \mathbb{C}$-linear self-adjoint and positive, $\mathrm{A} \mathbb{C}$-antilinear.

Then $\mathrm{L}$ and $\mathrm{A}$ commute, there exist a conjugation $\mathrm{c}$ commuting with $\mathrm{L}$ and $\mathrm{A}$ such that $\mathrm{Ac}$ is self-adjoint and non-negative and

$$
\mathrm{T}=\mathrm{e}^{\mathrm{c} \rho}
$$

with $\rho=\arg \cosh \mathrm{L}=\arg \sinh (\mathrm{Ac})$ a Hilbert-Schmidt, non-negative and self-adjoint operator commuting with c.

Proof. As $\mathrm{AA}^{*} \in \mathcal{L}_{1}(\mathcal{Z}), \mathrm{AA}^{*}=\sum_{j} \lambda_{j}^{2}\left|e_{j}\right\rangle\left\langle e_{j}\right|$, with $\lambda_{j} \in \mathbb{R}$ and $\sum_{j} \lambda_{j}^{2}<\infty$, from $\mathrm{L}^{2}=\mathrm{I}_{\mathcal{Z}}+\mathrm{A} \mathrm{A}^{*}$ we deduce $L^{2}=\sum_{j} \mu_{j}^{2}\left|e_{j}\right\rangle\left\langle e_{j}\right|$ with $\mu_{j}=\sqrt{1+\lambda_{j}^{2}}$ and thus $L=\sum_{j} \mu_{j}\left|e_{j}\right\rangle\left\langle e_{j}\right|$.

From the equivalent characterizations of a symplectomorphism we get

$$
\mathrm{L}^{2}-\mathrm{AA}^{*}=\mathrm{I}_{\mathcal{Z}} \quad \text { and } \quad \mathrm{L}^{2}-\mathrm{A}^{*} \mathrm{~A}=\mathrm{I}_{\mathcal{Z}}
$$

multiplying the first equality on the right and the second on the left by $A$ and computing the difference we get $\left[\mathrm{L}^{2}, A\right]=0$. As $\mathrm{L}$ is self-adjoint and positive one can use the functional calculus and $L=\sqrt{L^{2}}$ to obtain $[L, A]=0$.

From $[L, A]=0, L=L^{*}$ and the characterizations of a symplectomorphism, we also get $A L=$ $L A=L^{*} A=A^{*} L$ so that $\left(A-A^{*}\right) L=0$ and from the invertibility of $L$ one deduces $A=A^{*}$.

The proper subspaces associated with $\mathrm{L}$ and $\operatorname{ker}\left(\mathrm{L}-\mu \mathrm{I}_{\mathcal{Z}}\right)$, are thus stable by the action of $A$ (and finite-dimensional). We also remark that on $\operatorname{ker}\left(\mathrm{L}-\mu \mathrm{I}_{\mathcal{Z}}\right), A A^{*}=\mathrm{L}^{2}-\mathrm{I}_{\mathcal{Z}}=\left(\mu^{2}-1\right) \mathrm{I}_{\mathcal{Z}}$, so that two cases are possible:

$$
\mu=1, \text { then } A=0 \quad \text { or } \quad \mu>1, \text { then } \frac{1}{\sqrt{\mu^{2}-1}} A \frac{1}{\sqrt{\mu^{2}-1}} A^{*}=I_{\mathcal{Z}} .
$$

We apply Lemma A.1 to the $\mathbb{C}$-antilinear applications induced by the applications $A / \sqrt{\mu^{2}-1}$ on the Hilbert spaces $\operatorname{ker}\left(\mathrm{L}-\mu \mathrm{I}_{\mathcal{Z}}\right)$. This provides us with a Hilbert basis $\left(e_{j}\right)$ of $\mathcal{Z}$ which diagonalizes both $L$ and $A$. We can also define a conjugation $c\left(\sum_{j} \alpha_{j} e_{j}\right)=\sum_{j} \bar{\alpha}_{j} e_{j}$. This conjugation commutes with $L$ and $A$, and $A c$ is clearly a non-negative self-adjoint operator and so is necessarily $\sqrt{A A^{*}}$. We finally get for every vector $e_{j}$ of the basis the relations $L e_{j}=\mu_{j} e_{j}$ and $A e_{j}=\lambda_{j} e_{j}$ 
with $\mu_{j}^{2}-\lambda_{j}^{2}=1$, and thus one can define $\rho_{j}=\arg \cosh \mu_{j}\left(\rho_{j}=\arg \sinh \lambda_{j}\right.$ as $\left.\lambda_{j} \geq 0\right)$ and so we can define $\rho=\arg \cosh L=\arg \sinh A c$ so that $T=e^{c \rho}$.

\section{B Relations between Weyl and Wick symbols in finite di- mension}

We want to use the relation between the Weyl and Wick symbols associated to a same Wick polynomial in finite dimension, working with $\mathcal{Z}=\mathbb{C}^{\mathrm{r}}$ we have

$$
\mathrm{b}=\frac{1}{(\pi \varepsilon / 2)^{\mathrm{r}}} \breve{\mathrm{b}} * e^{-\frac{|z|^{2}}{\varepsilon / 2}}
$$

where $b$ is the Wick symbol and $\breve{b}$ is the Weyl symbol and $b^{\text {Wick }}=\breve{b}^{\text {Weyl }}$. We want to get rid of the convolution and for this we use the Fourier transform

$$
\mathcal{F} f\left(x^{\prime}\right)=\frac{1}{(2 \pi)^{r}} \int_{\mathbb{R}^{2 r}} e^{-i x \cdot x^{\prime}} f(x) d x
$$

where $x, x^{\prime} \in \mathbb{R}^{2 r} \cong \mathbb{C}^{r}$. The inverse Fourier transform is then

$$
\mathcal{F}^{-1} f(x)=\frac{1}{(2 \pi)^{r}} \int_{\mathbb{R}^{2 r}} e^{i x \cdot x^{\prime}} f\left(x^{\prime}\right) d x^{\prime} .
$$

We can use the formulae

$$
\begin{aligned}
\mathcal{F}(\mathrm{f} * \mathrm{~g}) & =(2 \pi)^{\mathrm{r}} \mathcal{F} \mathrm{f} . \mathcal{F} \mathrm{g} \\
\mathcal{F}\left[\mathrm{e}^{-\alpha \frac{|x|^{2}}{2}}\right]\left(x^{\prime}\right) & =\frac{1}{\alpha^{\mathrm{r}}} e^{-\frac{\left|x^{\prime}\right|^{2}}{2 \alpha}} \\
\mathcal{F}^{-1}(x \times \cdot) \mathcal{F} & =\mathrm{D}_{x} .
\end{aligned}
$$

We then obtain with $\mathrm{m}=2 \mathrm{n}$

$$
\begin{aligned}
\mathcal{F} \mathfrak{b}\left(z^{\prime}\right) & =\frac{(2 \pi)^{r}}{(\pi \varepsilon / 2)^{r}} \mathcal{F}\left[e^{-\frac{|z|^{2}}{\varepsilon / 2}}\right] \mathcal{F} \breve{b}\left(z^{\prime}\right) \\
& =\left(\frac{4}{\varepsilon}\right)^{r}\left(\frac{\varepsilon}{4}\right)^{r} e^{-\frac{\varepsilon}{8}\left|z^{\prime}\right|^{2}} \mathcal{F} \breve{b}\left(z^{\prime}\right) \\
& =e^{-\frac{\varepsilon}{8}\left|z^{\prime}\right|^{2}} \mathcal{F} \breve{b}\left(z^{\prime}\right)
\end{aligned}
$$

and

$$
\begin{aligned}
\mathrm{b} & =\mathcal{F}^{-1} e^{-\frac{\varepsilon}{8}\left|z^{\prime}\right|^{2}} \mathcal{F} \breve{b} \\
& =e^{-\frac{\varepsilon}{8} \mathcal{F}^{-1}\left|z^{\prime}\right|^{2} \mathcal{F} \breve{b}} \\
& =e^{\frac{\varepsilon}{2} \partial_{z} \cdot \partial_{\bar{z}} \breve{b}}
\end{aligned}
$$


using the fact that

$$
\mathcal{F}^{-1}\left|z^{\prime}\right|^{2} \mathcal{F}=D_{(x, \xi)}^{2}=-4 \times \frac{1}{2}\left(\partial_{x}-i \partial_{\xi}\right) \cdot \frac{1}{2}\left(\partial_{x}+i \partial_{\xi}\right)=-4 \partial_{z} \cdot \partial_{\bar{z}} .
$$

It is clear that if $\breve{b}$ is a polynomial in $\mathcal{P}_{\leq m}(\mathcal{Z})$, then $\mathrm{b}$ is in this class of polynomials, as we can see deriving the convolution product. We want to show that the map

$$
\begin{aligned}
\mathcal{P}_{\leq m}(\mathcal{Z}) & \rightarrow \mathcal{P}_{\leq m}(\mathcal{Z}) \\
\breve{\mathrm{b}} & \mapsto \mathrm{b}=\frac{1}{(\pi \varepsilon / 2)^{n}} \breve{\mathrm{b}} * \mathrm{e}^{-\frac{|z|^{2}}{\varepsilon / 2}}
\end{aligned}
$$

is bijective. As the dimension of $\mathcal{Z}$ is finite, the dimension of $\mathcal{P}_{\leq \mathrm{m}}(\mathcal{Z})$ is finite and it is enough to show that this map is one to one. For this we want to justify that on the part of main degree this application is the identity. This is obvious from the following facts:

- $\partial_{\bar{z}}^{\mathrm{q}} \partial_{z}^{\mathrm{p}} \mathrm{b}=\frac{1}{(\pi \varepsilon / 2)^{r}} \partial_{\bar{z}}^{\mathrm{q}} \partial_{z}^{\mathrm{p}} \breve{\mathrm{b}} * e^{-\frac{|z|^{2}}{\varepsilon / 2}}$

- this application is the identity on the constants.

Thus we can also consider the reverse application that we will improperly note

$$
\breve{\mathrm{b}}=\mathrm{e}^{-\frac{\varepsilon}{2} \partial_{z} \cdot \partial_{\bar{z}}} \mathrm{~b} .
$$

\section{Symplectic Fourier transform}

Let us then consider the symplectic Fourier transform on $\mathrm{L}^{2}\left(\mathbb{C}^{\mathrm{d}} ; \mathbb{C}\right) \equiv \mathrm{L}^{2}\left(\mathbb{R}^{2 \mathrm{~d}}\right)$ with $z=x+i \mathrm{y}$, defined by

$$
\mathcal{F}^{\sigma}(f)(z)=\int e^{i 2 \pi \sigma\left(z, z^{\prime}\right)} f\left(z^{\prime}\right) \mathrm{L}\left(\mathrm{d} z^{\prime}\right)
$$

with $\sigma\left(z, z^{\prime}\right)=\mathfrak{I}\left\langle z, z^{\prime}\right\rangle=\mathfrak{I}\left[\left\langle x, x^{\prime}\right\rangle+\left\langle y, y^{\prime}\right\rangle+\mathfrak{i}\left\langle x, y^{\prime}\right\rangle-\mathfrak{i}\left\langle y, x^{\prime}\right\rangle\right]$ and $L$ denotes the Lebesgue measure. We list here some properties of the symplectic Fourier transform.

(1) Inverse.

$$
\left(\mathcal{F}^{\sigma}\right)^{-1}=\mathcal{F}^{\sigma}
$$

(2) Convolution.

$$
\mathcal{F}^{\sigma}(f * g)=\mathcal{F}^{\sigma} f . \mathcal{F}^{\sigma} g
$$

(3) Composition with a symplectic transformation. Let T be a symplectomorphism, then

$$
\mathcal{F}^{\sigma}[\mathrm{f}(\mathrm{T} \cdot)](z)=\mathcal{F}^{\sigma}[\mathrm{f}](\mathrm{T} z) .
$$


(4) Gaussians. For $a>0$,

$$
\mathcal{F}^{\sigma}\left[e^{-a|\cdot|^{2}}\right](z)=\left(\frac{\pi}{a}\right)^{d} e^{-\pi^{2}|z|^{2} / a} .
$$

(5) Derivation. We consider the derivations $\partial_{z}=\frac{1}{2}\left(\partial_{x}-i \partial_{y}\right)$ and $\partial_{\bar{z}}=\frac{1}{2}\left(\partial_{x}+i \partial_{y}\right)$ then

$$
-\frac{1}{\pi} \partial_{z} \cdot z_{0}=\mathcal{F}^{\sigma}\left(\bar{z} \cdot z_{0} \times\right) \mathcal{F}^{\sigma} \text { and } \frac{1}{\pi} \bar{z}_{0} \cdot \partial_{\bar{z}}=\mathcal{F}^{\sigma}\left(\bar{z}_{0} \cdot z \times\right) \mathcal{F}^{\sigma} \text {. }
$$

\section{Acknowledgment}

The author would like to thank Francis Nier and Zied Ammari for profitable discussions.

$$
\text { Received: October 2011. Revised: November } 2011 .
$$

\section{References}

[1] Z. Ammari and F. Nier. Mean field limit for bosons and infinite dimensional phase-space analysis. Ann. Henri Poincaré,9(8):1503-1574, 2008.

[2] J. C. Baez, I. E. Segal, and Z.-F. Zhou. Introduction to algebraic and constructive quantum feld theory. Princeton Series in Physics. Princeton University Press, Princeton, NJ, 1992.

[3] F. A. Berezin. The method of second quantization. Academic Press, New York, 1966.

[4] F. A. Berezin and M. A. Shubin. The Schrödinger equation, volume 66 of Mathematics and its Applications (Soviet Series). Kluwer Academic Publishers Group, Dordrecht, 1991.

[5] L. Bruneau and J. Dereziński. Bogoliubov Hamiltonians and one-parameter groups of Bogoliubov transformations. J. Math. Phys., 48(2):022101, 24, 2007.

[6] M. Combescure, J. Ralston, and D. Robert. A proof of the Gutzwiller semiclassical trace formula using coherent states decomposition. Comm. Math. Phys., 202(2):463-480, 1999.

[7] M. Combescure and D. Robert. Semiclassical spreading of quantum wave packets and applications near unstable fixed points of the classical flow. Asymptot. Anal., 14(4):377-404, 1997.

[8] M. Combescure and D. Robert. Quadratic quantum Hamiltonians revisited. Cubo, 8(1):61-86, 2006.

[9] H. L. Cycon, R. G. Froese, W. Kirsch, and B. Simon. Schrödinger operators with application to quantum mechanics and global geometry. Texts and Monographs in Physics. Springer-Verlag, Berlin, study edition, 1987.

[10] W. G. Faris and R. B. Lavine. Commutators and self-adjointness of Hamiltonian operators. Comm. Math. Phys., 35:39-48, 1974. 
[11] J. Fröhlich, S. Graffi, and S. Schwarz. Mean-field- and classical limit of many-body Schrödinger dynamics for bosons. Comm. Math. Phys., 271(3):681-697, 2007.

[12] J. Ginibre and G. Velo. The classical field limit of scattering theory for nonrelativistic manyboson systems. I. Comm. Math. Phys., 66(1):37-76, 1979.

[13] J. Ginibre and G. Velo. The classical field limit of scattering theory for nonrelativistic manyboson systems. II. Comm. Math. Phys., 68(1):45-68, 1979.

[14] J. Ginibre and G. Velo. The classical field limit of nonrelativistic bosons. I. Borel summability for bounded potentials. Ann. Physics, 128(2):243-285, 1980.

[15] J. Ginibre and G. Velo. The classical field limit of nonrelativistic bosons. II. Asymptotic expansions for general potentials. Ann. Inst. H. Poincaré Sect. A (N.S.), 33(4):363-394, 1980.

[16] M. G. Grillakis, M. Machedon, and D. Margetis. Second-order corrections to mean field evolution of weakly interacting bosons. I. Comm. Math. Phys., 294(1):273-301, 2010.

[17] G. A. Hagedorn. Raising and lowering operators for semiclassical wave packets. Ann. Physics, 269(1):77-104, 1998.

[18] G. A. Hagedorn and A. Joye. Semiclassical dynamics with exponentially small error estimates. Comm. Math. Phys., 207(2):439-465, 1999.

[19] G. A. Hagedorn and A. Joye. Exponentially accurate semiclassical dynamics: propagation, localization, Ehrenfest times, scattering, and more general states. Ann. Henri Poincaré, $1(5): 837-883,2000$.

[20] K. Hepp. Phys., The classical limit for quantum mechanical correlation functions. Comm. Math. 35:265-277, 1974.

[21] E. Hille. Lectures on ordinary differential equations. Addison-Wesley Publ. Co., Reading, Mass.-London-Don Mills, Ont., 1969.

[22] L. Hörmander. Symplectic classifcation of quadratic forms, and general Mehler formulas. Math. Z., 219(3):413-449, 1995.

[23] T. Kato. Linear evolution equations of "hyperbolic" type. J. Fac. Sci. Univ. Tokyo Sect. I, 17:241-258, 1970.

[24] J. Kisyński. Sur les opérateurs de Green des problmes de Cauchy abstraits. Studia Math., 23:285-328, 1963/1964.

[25] T. Paul and A. Uribe. A construction of quasi-modes using coherent states. Ann. Inst. H. Poincaré Phys. Théor., 59(4):357-381, 1993.

[26] L. Polley, G. Reents, and R. F. Streater. Some covariant representations of massless boson fields. J. Phys. A, 14(9):2479-2488, 1981. 
[27] M. Reed and B. Simon. Methods of modern mathematical physics. II. Fourier analysis, selfadjointness. Academic Press [Harcourt Brace Jovanovich Publishers], New York, 1975.

[28] I. Rodnianski and B. Schlein. Quantum fuctuations and rate of convergence towards mean field dynamics. Comm. Math. Phys., 291(1):31-61, 2009.

[29] D. Shale. Linear symmetries of free boson fields. Trans. Amer. Math. Soc., 103:149-167, 1962. 